\title{
Neutrino oscillation and expected event rate of supernova neutrinos in adiabatic explosion model
}

\author{
Shio Kawagoe* \\ Knowledge Dissemination Unit, Institute of Industrial Science, The University of Tokyo, \\ 4-6-1 Komaba, Meguro-ku, Tokyo 153-8505, Japan \\ Division of Theoretical Astronomy, National Astronomical Observatory of Japan, \\ 2-21-1 Osawa, Mitaka-shi, Tokyo 181-8588, Japan \\ Takashi Yoshida \\ Department of Astronomy, School of Science, the University of Tokyo, \\ 7-3-1 Hongo, Bunkyo-ku, Tokyo, 113-0033, Japan \\ Toshitaka Kajino \\ National Astronomical Observatory of Japan, \\ 2-21-1 Osawa, Mitaka-shi, Tokyo 181-8588, Japan, \\ Department of Astronomy, School of Science, the University of Tokyo, \\ 7-3-1 Hongo, Bunkyo-ku, Tokyo, 113-0033, Japan \\ Hideyuki Suzuki \\ Department of Physics, Faculty of Science and Technology, Tokyo University of Science, \\ 2641 Yamazaki, Noda-shi, Chiba 278-8510, Japan \\ Kohsuke Sumiyoshi \\ Numazu College of Technology, \\ 3600 Ooka, Numazu-shi, \\ Shizuoka 410-8501, Japan \\ Shoichi Yamada \\ Department of Physics, Faculty of Science and Engineering, Waseda University, \\ 3-4-1 Okubo, Shinjuku-ku, Tokyo 169-8555, Japan
}

(Dated: November 22, 2017) 


\begin{abstract}
We study how the influence of the shock wave appears in neutrino oscillations and the neutrino spectrum using density profile of adiabatic explosion model of a core-collapse supernova which is calculated in an implicit Lagrangian code for general relativistic spherical hydrodynamics. We calculate expected event rates of neutrino detection at Super-Kamiokande and SNO for various $\theta_{13}$ values and both normal and inverted hierarchies. The predicted event rates of $\bar{\nu}_{e}$ and $\nu_{e}$ depend on the mixing angle $\theta_{13}$ for the inverted and normal mass hierarchies, respectively, and the influence of the shock wave appears for about $2-8 \mathrm{~s}$ when $\sin ^{2} 2 \theta_{13}$ is larger than $10^{-3}$. These neutrino signals for the shock wave propagation is decreased by $\lesssim 30 \%$ for $\bar{\nu}_{e}$ in inverted hierarchy (SK) or by $\lesssim 15 \%$ for $\nu_{e}$ in normal hierarchy (SNO) compared with the case without shock. The obtained ratio of the total event for high-energy neutrinos $\left(20 \mathrm{MeV} \leqq E_{\nu} \leqq 60 \mathrm{MeV}\right)$ to low-energy neutrinos ( $5 \mathrm{MeV} \lesssim E_{\nu} \leqq 20 \mathrm{MeV}$ ) is consistent with the previous studies in schematic semi-analytic or other hydrodynamic models of the shock propagation. The time dependence of the calculated ratio of the event rates of high-energy neutrinos to the event rates of low-energy neutrinos is a very useful observable which is sensitive to $\theta_{13}$ and mass hierarchies. Namely, time-dependent ratio shows clearer signal of the shock wave propagation that exhibits remarkable decrease by at most factor $\sim 2$ for $\bar{\nu}_{e}$ in inverted hierarchy (SK), whereas it exhibits smaller change by $\sim 10 \%$ for $\nu_{e}$ in normal hierarchy (SNO). Observing time-dependent high-energy to low-energy ratio of the neutrino events thus would provide a piece of very useful information to constrain $\theta_{13}$ and mass hierarchy, and eventually help understanding the propagation how the shock wave propagates inside the star.
\end{abstract}

PACS numbers: Valid PACS appear here

*The University of Tokyo; Electronic address: shiok@iis.u-tokyo.ac.jp 


\section{INTRODUCTION}

About twenty events of supernova neutrinos were detected from SN1987A [1, 2]. Some neutrino features such as neutrino temperatures and the energy carried out by neutrinos that had been obtained from the data of SN1987A were consistent with theoretical expectation [3 6]. However, the detected number of the neutrino events was still too small to find out more details about other flavors of the supernova neutrinos and the explosion mechanism. Although, we have not yet obtained neutrino events from the second core-collapse supernova which is the rare event of the century, detection of next supernova neutrinos is highly desirable to obtain important information on the neutrinos and the explosion mechanism. The expected information from the supernova neutrino observations is classified into two categories of implication for neutrino physics and for supernova physics.

Large-volume underground detectors are now operating to detect various neutrino events. For example, Super-Kamiokande (SK), which is at the Kamioka mine in Japan, is a water Cherenkov detector, filled with 50,000 ton pure water (32,000 ton fiducial volume for the burst mode and 22,500 ton for the other modes) [7]. KamLAND is a liquid scintillator detector with 1,000 ton active volume [8]. Sudbury Neutrino Observatory (SNO) has operated as a heavy water Cherenkov detector, filled with 1,000 ton fiducial volume [9]. Now, a new generation experiment, $\mathrm{SNO}+$, is planned to be constructed [10]. We achieved remarkable development of the neutrino physics, especially about neutrino oscillation from the solar, atmospheric and reactor neutrino experiments with these detectors. In addition, the supernova neutrinos are thought to be fascinating targets of these detectors. If one supernova explodes at the Galactic center, thousands of neutrino events will be observed in Super-Kamiokande [11]. Furthermore, megaton-size neutrino detectors will detect $\times 10^{5}$ supernova neutrino events in the future [12-16]. The neutrinos are released directly from the core, in which the extreme physical condition of very high density is realized. Therefore, the supernova neutrinos would become the probe of such an environment.

Neutrino flavor change by the neutrino oscillations relates to neutrino oscillation parameters, i.e., the mixing angles, mass hierarchy, and CP violation phase. Most of neutrino oscillation parameters have been determined by the various neutrino experiments [17-19]. However, the mixing angle $\theta_{13}$ is not precisely constrained, and only the upper bound is known from reactor experiments (e.g., [20]). In addition, the neutrino mass hierarchy, i.e., 
the sign of $\Delta m_{13}^{2} \equiv m_{3}^{2}-m_{1}^{2}$, and the $\mathrm{CP}$ violation phase remain unknown. However, among many studies about implication on these unknown neutrino parameters from future supernova neutrinos [21 29], there are several proposed possibilities that the detection of the neutrinos from the next Galactic supernova would constrain the neutrino oscillation parameters and identify the mass hierarchy more precisely [26-29].

Most of the supernova neutrinos are released for about 10 seconds after the core bounce, and interact with electrons when the neutrinos propagate through stellar matter. Therefore, Mikheyev-Smirnov-Wolfstein (MSW) effect is to be taken into account in the neutrino oscillations of the supernova neutrinos. The MSW effect on the supernova neutrino signal has been investigated previously in literatures (e.g., [21, 30 32]). For example, the initial progenitor mass dependence of the early neutrino burst taking account of the MSW effect was studied in [33], and the Earth matter effects of the supernova neutrinos were identified in [34-37]. Moreover, neutrino spin-flavor conversion in supernova has been studied considering numerically in [38-40] and analytically in [41, 42].

Recently, the effect of the shock wave on MSW effect of neutrino oscillations in supernova was studied [22, 43]. This effect appears as a decrease of the average energy of $\nu_{e}$ in the case of normal mass hierarchy (or $\bar{\nu}_{e}$ in the case of inverted mass hierarchy) [44, 45]. Since the shock wave passes through H-resonance region, whose typical resonance density is $10^{3} \mathrm{~g} / \mathrm{cm}^{3}$ in a few seconds after core bounce, the adiabaticity of the resonance changes. The time dependence of the neutrino events monitors the density profile of the supernova [26 28, 45, 46]. Therefore, using MSW effects embedded in the supernova neutrinos, we are able to find the density profile of the supernova. However, in many previous studies the influences of the shock wave on the neutrino events were discussed schematically using simplified and parameterized shock-wave profiles [27].

There are many supernova simulations taking account of various explosion mechanisms. However, there are a few studies in which the neutrino oscillations were calculated by using simulation results because almost all supernova simulations were performed in only core region although MSW effect occurs at outer envelope of the star. In practice, it is still very hard to carry out multidimensional simulation with neutrino transport to proceed to the shock propagation from the core throughout the envelope. Therefore, it is important for the studies of the neutrino oscillations to calculate the shock propagation throughout a supernova not only in the core but also in the envelope. 
In this paper, we study how the shock wave propagation and the neutrino oscillation parameters affect the supernova neutrinos. We calculate neutrino oscillations of the supernova neutrinos by using supernova simulation result in adiabatic explosion model. In our model, we calculate all processes of core collapse, bounce, and shock propagation continuously in a unified manner. In order to clarify detailed dependence of the result on the neutrino oscillation parameters and the shock wave, we calculate survival probabilities, energy spectra, and the event rates of three flavors of supernova neutrinos to be detected with SK and SNO.

In Sec. III, we introduce our numerical method. In Sec. III, we show time evolution of the survival probability and energy spectra of the supernova neutrinos. We also study the event rates. We analyze the dependence of these neutrino signals on neutrino oscillation parameters and the shock effect. We discuss the ratio of high- to low-energy neutrino events which will be detected by SK and SNO. We discuss the difference of the expected supernova neutrinos using both parameterized density and the density obtained by the simulations of supernova explosion in Sec. IIIE. We also discuss other effects of supernova explosions and supernova neutrinos which should affect the time evolution of the neutrino signal. Finally, we conclude our paper in Sec. IV.

\section{NUMERICAL METHOD}

\section{A. Supernova Model}

In order to obtain the detailed density profile in a supernova explosion and to examine the MSW effect on supernova neutrinos, we use one dimensional simulation result of the supernova. We model the supernova explosion using an implicit Lagrangian code for general relativistic spherical hydrodynamics [47]. The numerical tables of Shen's relativistic equation of state (EOS) [48] and Timmes's EOS [49] are adopted for the high and low density matters in this code, respectively. We adopt the presupernova model of a $15 M_{\odot}$ star provided by Woosley and Weaver (WW95) [50] as the initial condition. We use the distribution of the electron fraction $Y_{e}$ in WW95. We perform calculations of all processes throughout the core collapse, bounce, and shock propagation continuously by adiabatic collapse with fixed electron fraction to follow the shock wave for a long time scale $(>10 \mathrm{~s})$ and a wide density

range, consistently [51]. We thus succeeded in the calculation of the propagation of the 
shock, which is generated by the collapse of the iron core, until the shock wave reaches the hydrogen rich envelope. Throughout one calculation, one finds that the density of the central core reaches $\sim 10^{15} \mathrm{~g} / \mathrm{cm}^{3}$ and the shock wave propagation for beyond the hydrogen envelope $\left(\sim 1 \mathrm{~g} / \mathrm{cm}^{3}\right)$ is solved simultaneously [52, 53$]$.

Figure 1 shows the calculated density profiles as a function of radius for every $1 \mathrm{~s}$ from 0 to $7 \mathrm{~s}$ after the core bounce. The shock wave reaches the oxygen-rich layer in $1 \mathrm{~s}$ and passes through the helium layer in $10 \mathrm{~s}$. The horizontal lines show the density at the H-resonance of the neutrino energy. The shock wave reaches the H-resonance in about $2 \mathrm{~s}$. The density behind the shock wave hardly decreases because we neglected neutrino cooling of the protoneutron star in the present study. Therefore, the shock velocity is kept almost constant at $9 \times 10^{8} \mathrm{~cm} / \mathrm{s}$ in this model. When the neutrino cooling of the proto-neutron star is taken into consideration, the density and the pressure behind the shock would inevitably decrease. As a result, the shock wave would be decelerated. We study detailed analysis of the cooling effect of the proto-neutron star elsewhere.

We assume the neutrino energy spectra with Fermi-Dirac distributions. Numerical simulations of supernova explosions which include neutrino transport suggested that the resultant neutrino spectra at low-to-intermediate energies do not exactly follow Fermi-Dirac distributions. However, since we are interested in the event number of neutrinos to be detected in a water Cherenkov detector, the neutrino signals are sensitive to high energy neutrinos because the neutrino-induced reaction cross sections in the detector are proportional to the neutrino energy. As large as the neutrino energies above $\sim 10 \mathrm{MeV}$ are concerned, therefore, FermiDirac distributions with finite chemical potentials may be justified as one of the reasonable approximations to the results of numerical simulations of the neutrino transport ([54]; and references therein). Although several simulations suggest different neutrino temperatures from one another, they all satisfy a common hierarchy $T_{\nu_{e}}<T_{\bar{\nu}_{e}}<T_{\nu_{x}}$. The interactions between $\nu_{e}$ and neutrons freeze out at lower temperature and density than the interactions between $\bar{\nu}_{e}$ and protons do inside the neutralized supernova core, while the interactions between $\nu_{x}\left(=\nu_{\mu}, \bar{\nu}_{\mu}, \nu_{\tau}\right.$, and $\left.\bar{\nu}_{\tau}\right)$ and nucleons freeze out at much higher temperature and density because $\nu_{x}$ interact with matter only through the neutral current. In the present study, the neutrino temperatures are set to be $T_{\nu_{e}}=3.5 \mathrm{MeV}, T_{\bar{\nu}_{e}}=4 \mathrm{MeV}$, and $T_{\nu_{x}}=7$ $\mathrm{MeV}$ associated with the neutrino chemical potentials $\mu_{\nu_{e}}=7.28 \mathrm{MeV}, \mu_{\bar{\nu}_{e}}=10 \mathrm{MeV}$, and $\mu_{\nu_{x}}=0 \mathrm{MeV}$. These parameter values are well approximated to the time integrated spectra 
of the neutrinos obtained from a supernova simulation in Livermore Group [11]. Previous studies of energy spectra of supernova neutrinos in Galactic chemical evolution of ${ }^{11} \mathrm{~B}$ and ${ }^{10} \mathrm{~B}[55,56]$ and the r-process nucleosynthesis [57, 58] indicated that the neutrino temperatures are constrained by the theoretical fit to the observed elemental abundances of these nuclei : $T_{\nu_{e}}=2.5-3.5 \mathrm{MeV}, T_{\bar{\nu}_{e}}=4-5 \mathrm{MeV}$, and $T_{\nu_{x}}=4-7 \mathrm{MeV}$, still satisfying hierarchy condition $T_{\nu_{e}}<T_{\bar{\nu}_{e}}<T_{\nu_{x}}$, where the uncertainty due to finite chemical potentials [55] are taken into account in the inferred temperature values. Our adopted values $T_{\nu_{e}}=3.5$ $\mathrm{MeV}, T_{\bar{\nu}_{e}}=4 \mathrm{MeV}$, and $T_{\nu_{x}}=7 \mathrm{MeV}$ are in reasonable agreement with these constraints. The energy spectrum of each flavor of neutrinos is presumed to be independent of time. The total neutrino energy carried by neutrinos is set equal to $3 \times 10^{53}$ erg and the energy is equipartitioned to three-flavors of neutrinos and anti-neutrinos. We assume the exponential decay of the neutrino luminosity with a decay time of $3 \mathrm{~s}$.

\section{B. Neutrino Oscillation}

In order to calculate neutrino oscillation effect on the neutrino spectrum, we solve the time evolution of the neutrino wave function along the density profile of our supernova model. The time evolution of the neutrino wave function is solved using the equation

$$
\imath \frac{d}{d t}\left(\begin{array}{c}
\nu_{e} \\
\nu_{\mu} \\
\nu_{\tau}
\end{array}\right)=\left\{U\left(\begin{array}{ccc}
0 & 0 & 0 \\
0 & \Delta E_{12} & 0 \\
0 & 0 & \Delta E_{13}
\end{array}\right) U^{-1}+\left(\begin{array}{ccc}
\sqrt{2} G_{\mathrm{F}} n_{e}(r) & 0 & 0 \\
0 & 0 & 0 \\
0 & 0 & 0
\end{array}\right)\right\}\left(\begin{array}{c}
\nu_{e} \\
\nu_{\mu} \\
\nu_{\tau}
\end{array}\right)
$$

where $\Delta E_{i j}=\Delta m_{i j}^{2} / 2 E_{\nu}, \Delta m_{i j}^{2} \equiv m_{j}^{2}-m_{i}^{2}, G_{F}, E_{\nu}$ and $n_{e}(r)$ are the energy difference between two mass eigenstates, mass squared differences, the Fermi constant, the neutrino energy, and the electron number density. In case of anti-neutrinos, the sign of $\sqrt{2} G_{F} n_{e}$ changes. $U$ is the Cabibbo-Kobayashi-Masukawa (CKM) matrix

$$
U=\left(\begin{array}{ccc}
c_{12} c_{13} & s_{12} c_{13} & s_{13} e^{-i \delta} \\
-s_{12} c_{23}-c_{12} s_{23} s_{13} e^{i \delta} & c_{12} c_{23}-s_{12} s_{23} s_{13} e^{i \delta} & s_{23} c_{13} \\
s_{12} s_{23}-c_{12} c_{23} s_{13} e^{i \delta} & -c_{12} s_{23}-s_{12} c_{23} s_{13} e^{i \delta} & c_{23} c_{13}
\end{array}\right)
$$

where $s_{i j}=\sin \theta_{i j}$, and $c_{i j}=\cos \theta_{i j}(i \neq j=1,2,3)$ [59]. We here put the CP violating phase $\delta$ equal to zero in the CKM matrix. 
The neutrino oscillation parameters are taken from the analysis of various neutrino experiments [17, 18], except for $\theta_{13}$ [19], as

$$
\sin ^{2} 2 \theta_{12}=0.84, \quad \sin ^{2} 2 \theta_{23}=1.00
$$

and

$$
\Delta m_{12}^{2}=8.1 \times 10^{-5} \mathrm{eV}^{2}, \quad\left|\Delta m_{13}^{2}\right|=2.2 \times 10^{-3} \mathrm{eV}^{2} .
$$

We calculate the neutrino survival probabilities for both normal $\left(\Delta m_{13}^{2}>0\right)$ and inverted $\left(\Delta m_{13}^{2}<0\right)$ mass hierarchies for four cases of $\sin ^{2} 2 \theta_{13}$ value: $\sin ^{2} 2 \theta_{13}=10^{-2}, 10^{-3}, 10^{-4}$ and $10^{-5}$.

The energy spectra of the neutrinos passed through the exploding supernova, $\phi_{\nu}^{\mathrm{SN}}\left(E_{\nu}\right)$, are obtained from the spectra of the neutrino emitted from the neutrino sphere, $\phi_{\nu}^{\text {org }}\left(E_{\nu}\right)$, multiplied by the survival probability in accordance with the following equation,

$$
\left(\begin{array}{c}
\phi_{\nu_{e}}^{\mathrm{SN}}\left(E_{\nu}\right) \\
\phi_{\bar{\nu}_{e}}^{\mathrm{SN}}\left(E_{\nu}\right) \\
\phi_{\nu_{x}}^{\mathrm{SN}}\left(E_{\nu}\right)
\end{array}\right)=\left(\begin{array}{ccc}
P\left(E_{\nu}\right) & 0 & 1-P\left(E_{\nu}\right) \\
0 & \bar{P}\left(E_{\nu}\right) & 1-\bar{P}\left(E_{\nu}\right) \\
1-P\left(E_{\nu}\right) & 1-\bar{P}\left(E_{\nu}\right) & 2+P\left(E_{\nu}\right)+\bar{P}\left(E_{\nu}\right)
\end{array}\right)\left(\begin{array}{c}
\phi_{\nu_{e}}^{\mathrm{org}}\left(E_{\nu}\right) \\
\phi_{\bar{\nu}_{e}}^{\mathrm{org}}\left(E_{\nu}\right) \\
\phi_{\nu_{x}}^{\mathrm{org}}\left(E_{\nu}\right)
\end{array}\right),
$$

where $\phi_{\nu_{x}} \equiv \frac{1}{4}\left(\phi_{\nu_{\mu}}+\phi_{\nu_{\tau}}+\phi_{\bar{\nu}_{\mu}}+\phi_{\bar{\nu}_{\tau}}\right)$, and $P$ and $\bar{P}$ are survival probabilities of $\nu_{e}$ and $\bar{\nu}_{e}$, respectively.

Neutrinos change largely their flavors at resonances of neutrino oscillations. There are two resonances for supernova neutrinos (e.g., [24]). The resonance points of higher and lower electron number densities correspond to H-resonance and L-resonance, respectively. The electron number density at the resonance point is written as

$$
n_{e, r e s} \equiv \frac{1}{2 \sqrt{2} G_{F}} \frac{\Delta m^{2}}{E_{\nu}} \cos 2 \theta
$$

where $\Delta m^{2}$ and $\theta$ correspond to $\left|\Delta m_{13}^{2}\right|$ and $\theta_{13}$ at H-resonance and to $\Delta m_{12}^{2}$ and $\theta_{12}$ at L-resonance, respectively. The flavor change at a resonance strongly depends on adiabaticity of the resonance. When the resonance is adiabatic, large flavor change occurs. The neutrino adiabaticity is estimated by $\gamma$,

$$
\gamma \equiv \frac{\Delta m^{2}}{2 E_{\nu}} \frac{\sin ^{2} 2 \theta}{\cos 2 \theta} \frac{n_{e, r e s}}{\left|\frac{d n_{e}}{d r}\right|} .
$$


If $\gamma$ is larger than 1, the resonance becomes adiabatic, but if it is small, resonance becomes non-adiabatic. The change of adiabaticity mainly occurs at H-resonance owing to the shock propagation. When the shock wave reaches the H-resonance, the density gradient becomes large and the value of $\gamma$ decreases. This leads to a change of survival probability of neutrinos. Since the resonance point is inversely proportional to the neutrino energy, this effect appears from low-energy side and moves toward high-energy side according to the shock wave propagation. As shock wave propagates outward, the density at the shock front decreases and the resonance condition is satisfied for higher energy neutrinos.

\section{Detection of Supernova Neutrinos}

The expected event number of the neutrinos in a water Cherenkov detector can be expressed as

$$
\frac{d^{2} N}{d E_{e} d t}=N_{t a r} \eta_{\left(E_{e}\right)} \frac{1}{4 \pi d^{2}} \frac{d^{2} N_{\nu}}{d E_{\nu} d t} \sigma\left(E_{\nu}\right) \frac{d E_{\nu}}{d E_{e}}
$$

where $N$ is the detection number of neutrinos, $E_{e}$ is the energy of electron or positron, $E_{\nu}$ is the energy of neutrino, $N_{t a r}$ is the target number, $\eta_{\left(E_{e}\right)}$ is the efficiency of the detector, $d$

is the distance from the supernova, $\frac{d^{2} N_{\nu}}{d E_{\nu} d t}$ is the neutrino spectrum, and $\sigma\left(E_{\nu}\right)$ is the cross section as a function of the neutrino energy [59]. We assumed a supernova at the center of Milky Way $(d=10 \mathrm{kpc})$, and we neglected the Earth effect [60]. We assume the detection at SK and SNO.

The mass of SK is 32000 ton and the solvent is pure water. SK mainly detects the $\bar{\nu}_{e}$ events with the reaction $\bar{\nu}_{e}+p \rightarrow e^{+}+n$. The event number is obtained by integrating over the angular distribution of the events. As for the efficiency of the detector, we assumed as follows for simplicity: $\eta_{\left(E_{e}\right)}=0$ for $E_{e}<7 \mathrm{MeV}$ and $\eta_{\left(E_{e}\right)}=1$ for $E_{e} \geq 7 \mathrm{MeV}$ in accordance with SK Phase II [61]. The finite energy resolution of the detector was neglected here. The $\bar{\nu}_{e}$ and $\nu_{e}$ events are evaluated taking account of the following four reactions,

$$
\begin{gathered}
\overline{\nu_{e}}+p \rightarrow e^{+}+n, \\
\nu_{e}+e^{-} \rightarrow \nu_{e}+e^{-}, \\
\overline{\nu_{e}}+e^{-} \rightarrow \overline{\nu_{e}}+e^{-}, \\
\nu_{x}+e^{-} \rightarrow \nu_{x}+e^{-} .
\end{gathered}
$$


The energies of positron and electron are evaluated as $E_{e^{+}}=E_{\nu}+m_{p}-m_{n}-m_{e+}$ for $\overline{\nu_{e}}+p \rightarrow n+e^{+}$and $E_{e}=E_{\nu}-\frac{m_{e}}{2}$ for $\nu+e^{-} \rightarrow \nu+e^{-}$, respectively. The cross sections of the above four reactions are adopted from [59].

The mass of SNO is 1000 ton and the solvent is heavy water. SNO detects not only $\bar{\nu}_{e}$ events but also $\nu_{e}$ events with charged and neutral current reactions. As for the efficiency of the detector, we assumed as follows for simplicity: $\eta_{\left(E_{e}\right)}=0$ for $E_{e}<5 \mathrm{MeV}$ and $\eta_{\left(E_{e}\right)}=1$ for $E_{e} \geq 5 \mathrm{MeV}$ [62]. We evaluate the $\bar{\nu}_{e}$ and $\nu_{e}$ event numbers using the four reactions,

$$
\begin{aligned}
& \nu_{e}+d \rightarrow p+p+e^{-} \\
& \bar{\nu}_{e}+d \rightarrow n+n+e^{+} \\
& \nu+d \rightarrow p+n+\nu \\
& \nu+e^{-} \rightarrow \nu+e^{-} .
\end{aligned}
$$

The main reactions of $\bar{\nu}_{e}$ and $\nu_{e}$ are the first and second reactions, respectively [63]. The cross sections of these reactions are adopted from [64]. The cross sections become larger as the neutrino energy increases. Although the SNO experiment has already ended, we also evaluate the neutrino events by SNO because the detection efficiency of $\nu_{e}$ is high and similar in next generation detector like SNO.

\section{RESULT AND DISCUSSIONS}

\section{A. Survival probability}

We calculate the survival probabilities of $\nu_{e}$ and $\bar{\nu}_{e}$ from the supernova, $P\left(E_{\nu}\right)$ and $\bar{P}\left(E_{\nu}\right)$. Figure 2 shows the calculated results. Left panels of Figure 2 are $P\left(E_{\nu}\right)$ in the case of normal hierarchy in every $1 \mathrm{~s}$, and right panels of Figure 2 are $\bar{P}\left(E_{\nu}\right)$ in the case of inverted hierarchy. Red, green, blue, purple, sky blue and orange lines correspond to the results in $t=0,1,2,3$, 4 and $5 \mathrm{~s}$ after core bounce. We showed the results for the values of $\sin ^{2} 2 \theta_{13}=10^{-2}, 10^{-3}$, $10^{-4}$ and $10^{-5}$ from top to bottom. In the case of $\sin ^{2} 2 \theta_{13}=10^{-2}$ and $10^{-3}, P\left(E_{\nu}\right)$ and $\bar{P}\left(E_{\nu}\right)$ are about 0 when shock wave does not reach H-resonance $(t=0-2 \mathrm{~s})$. However, in later time $(t=2-5 \mathrm{~s}), P\left(E_{\nu}\right)$ and $\bar{P}\left(E_{\nu}\right)$ become finite. These effects appear from low-energy side and gradually shift toward high-energy side as the time passes by. In the case of $\sin ^{2} 2 \theta_{13}=10^{-5}, P\left(E_{\nu}\right)$ and $\bar{P}\left(E_{\nu}\right)$ hardly change with time, and are about 0.3 and 
0.7 , respectively. In the case of $\sin ^{2} 2 \theta_{13}=10^{-4}, P\left(E_{\nu}\right)$ and $\bar{P}\left(E_{\nu}\right)$ are about 0.2 and 0.4 in $t=0-2 \mathrm{~s}$, respectively. These survival probabilities indicate intermediate adiabaticity between the cases for large $\theta_{13}$ and small $\theta_{13}$. At the later times they become large as shown in the large $\theta_{13}$ case.

We can understand these behaviors considering the shock wave propagation. The adiabaticity of H-resonance is estimated by $\gamma$ in Eq. (77). When the shock wave reaches H-resonance region, the adiabaticity changes. If $\sin ^{2} 2 \theta_{13}$ is large and there is not the shock wave at the resonance region, $\gamma$ is larger than 1 , and the resonance is adiabatic. As a result, the survival probabilities are 0 . When the shock wave reaches a resonance, large density gradient reduces $\gamma$ and the resonance becomes non-adiabatic. Thus, the survival probabilities become finite during the shock passage in the resonance region. In contrast, if $\sin ^{2} 2 \theta_{13}$ is very small, $\gamma$ is smaller than 1 , and the resonance is non-adiabatic. Consequently, the survival probabilities are finite, and the influence of the shock wave hardly appears to the survival probability, since H-resonance totally in non-adiabatic regardless the existence of the shock wave. In the case of $\sin ^{2} 2 \theta_{13}=10^{-2}$, however, the influence of the shock wave is not clearly seen though the $\sin ^{2} 2 \theta_{13}$ is large. This is because that $\sin ^{2} 2 \theta_{13}$ is very large and $\gamma$ is larger than 1 even when $\left|\frac{1}{n_{e}} \frac{d n_{e}}{d r}\right|$ becomes large.

$\gamma$ can be rewritten by the ratio of the length characterizing the neutrino oscillations $L_{\text {osc }}=\frac{2 E_{\nu}}{\Delta \tilde{m}^{2} \sin 2 \theta}$, where $\Delta \tilde{m}^{2}=\left\{\left(2 \sqrt{2} G_{F} E_{\nu} n_{e}(r) / \Delta m^{2}-\cos 2 \theta\right)^{2}+\sin ^{2} 2 \theta\right\}^{1 / 2} \Delta m^{2}$, to the length of the level crossing region, i.e. the level crossing length $\delta r=\frac{\sin 2 \theta}{\cos 2 \theta}\left|\frac{1}{n_{e}} \frac{d n_{e}}{d r}\right|^{-1}$ at resonance. Therefore, the adiabaticity of the neutrinos can be evaluated by $L_{\text {osc }}$ and $\delta r$ [59]. The resonance is adiabatic when the level crossing length is larger than the oscillation length, $L_{\text {osc }} \ll \delta r$. Figure 3 shows the level crossing region and the oscillation length of neutrinos $\left(E_{\nu}=5 \mathrm{MeV}\right)$ in the case of inverted mass hierarchy as a function of the radius. The left panels of Figure 3 are at $t=0 \mathrm{~s}$, and the right panels are at $t=3 \mathrm{~s}$, respectively. Shown are the results for $\sin ^{2} 2 \theta_{13}=10^{-2}, 10^{-3}, 10^{-4}$ and $10^{-5}$ from top to bottom. Red and green lines display the oscillation lengths of $\nu_{e}$ and $\bar{\nu}_{e}$, and blue line shows the level crossing length using $\theta_{13}$. The oscillation length of $\bar{\nu}_{e}$ becomes extremely large on the resonance, and its behavior depends on $\sin ^{2} 2 \theta_{13}$ below or above the resonance strongly. On the other hand, the oscillation length of $\nu_{e}$ changes rather gently as a function of radius, and strong dependence on $\sin ^{2} 2 \theta_{13}$ is not clearly seen. As $\sin ^{2} 2 \theta_{13}$ becomes smaller or the shock wave reaches the resonance, the level crossing length becomes shorter. 
In the left panels $(t=0 \mathrm{~s})$, the level crossing length at $\sin ^{2} 2 \theta_{13}=10^{-2}$ and $10^{-3}$ are larger than the oscillation length at all region. Therefore, the resonance is adiabatic. On the other hand, in the case of $\sin ^{2} 2 \theta_{13}=10^{-4}$ and $10^{-5}$, the level crossing length at the resonance is almost same as or slightly smaller than the oscillation length of $\bar{\nu}_{e}$. Therefore, the resonance is non-adiabatic. In the right panels $(t=3 \mathrm{~s})$ when the shock wave reaches the H-resonance, the level crossing length, $\delta r$, is of the same order or smaller than the oscillation length $L_{\mathrm{osc}}$ for all cases of the mixing angle, expect for $\sin ^{2} 2 \theta_{13}=10^{-2}$. This satisfies non-adiabatic condition $\gamma<1$ as discussed above. We understand, therefore, that the resonance becomes non-adiabatic by the effect of the shock wave. In Figure 2, the influence of the shock wave appears about $2 \mathrm{~s}$ after core bounce. This result is consistent with our simulation (see Figure 11).

In the case of normal hierarchy, the survival probability of $\bar{\nu}_{e}$ does not change much because there is no resonance in the $\bar{\nu}$ sector. Therefore, the survival probability of $\bar{\nu}_{e}$ in normal hierarchy is always $\sim 0.7$ regardless of the value of $\sin ^{2} 2 \theta_{13}$ or independently of the shock wave.

We note that there is L-resonance in $\nu$ sector even in the case of inverted hierarchy. However, the value of $\theta_{12}$ which is related to L-resonance is very large (see Eq. (3)), and the level crossing length of our simulation is not as small as the oscillation length at the resonance. Therefore, $\gamma$ at the L-resonance is larger than 1. As a result, the survival probability of $\nu_{e}$ in inverted hierarchy does not change drastically, and stays always $\sim 0.3$.

\section{B. Supernova neutrino spectrum}

We calculate the supernova neutrino spectra using the survival probabilities. Figure 4 shows the spectra of $\nu_{e}$. Left panels of Figure 4 show the spectra in the case of normal hierarchy, and right panels show the results of inverted hierarchy. Figure 5 is same as Figure 4 but for $\bar{\nu}_{e}$ spectra. Red solid and blue dotted lines are the spectra with and without shock wave, respectively. In order to clearly observe the shock wave effects, we display these ratio, $\phi_{\text {with }} / \phi_{\text {without }}$, of the spectra with to without shock in lower part of each panel.

We see clearly the shock wave effects in the $\nu_{e}$ spectra in Figure 4 in normal hierarchy. In the case of $\sin ^{2} 2 \theta_{13}=10^{-3}$ and $10^{-4}$, an enhancement in low energy component of the neutrino spectra is seen when the shock wave reaches the H-resonance. At later times, the 
influence of the shock wave on the spectra moves from the low-energy side to the high-energy side. The effect at later times is seen as a reduction of the high energy component of the neutrino spectrum. The influence of the shock wave in the case of $\sin ^{2} 2 \theta_{13}=10^{-2}$ does not clearly appear in spectra for the same reason as discussed in the previous section. In the case of $\sin ^{2} 2 \theta_{13}=10^{-5}$, the neutrino spectra with shock are not different from the spectra without shock. H-resonance is non-adiabatic even without the shock wave. We do not see any shock effects on the $\bar{\nu}_{e}$ spectra in inverted hierarchy.

From Figure 5, the effect of shock wave appears in the spectra of $\bar{\nu}_{e}$ in the case of inverted hierarchy. The dependence of the $\bar{\nu}_{e}$ spectra on $\sin ^{2} 2 \theta_{13}$ is almost the same as the dependence of the $\nu_{e}$ spectra in normal hierarchy.

The spectrum of $\nu_{e}$ at the surface of the supernova is written from Eq.(5) as

$$
\phi_{\nu_{e}}^{\mathrm{SN}}\left(E_{\nu}\right)=P\left(E_{\nu}\right)\left\{\phi_{\nu_{e}}^{\mathrm{org}}\left(E_{\nu}\right)-\phi_{\nu_{x}}^{\mathrm{org}}\left(E_{\nu}\right)\right\}+\phi_{\nu_{x}}^{\mathrm{org}}\left(E_{\nu}\right) .
$$

The initial spectra are $\phi_{\nu_{e}}^{\text {org }}>\phi_{\nu_{x}}^{\text {org }}$ in low energy side, and $\left(\phi_{\nu_{e}}^{\text {org }}-\phi_{\nu_{x}}^{\text {org }}\right)$ is positive. Therefore, $\phi_{\nu_{e}}^{\text {obs }}$ increases when $P$ is not 0 . On the other hand, the initial spectra are $\phi_{\nu_{e}}^{\text {org }}<\phi_{\nu_{x}}^{\text {org }}$ in

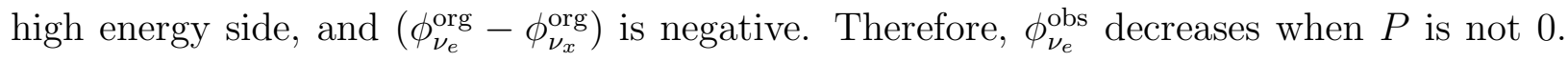
These increase and decrease appear in the observed neutrino spectra as the result from the influence of the shock wave. In the lower part of each panel, we can see this effect clearly. We note that the energy that satisfies $\phi_{\nu_{e}}^{\text {org }}=\phi_{\nu_{x}}^{\text {org }}$ is about $18.7 \mathrm{MeV}$, and the energy that satisfies $\phi_{\bar{\nu}_{e}}^{\text {org }}=\phi_{\nu_{x}}^{\text {org }}$ is about $23.3 \mathrm{MeV}$.

\section{Event rate of supernova neutrino}

We discuss here the predicted event rates of the supernova neutrinos to be detected with SK in the manner described in section [C] The upper parts of each panel of Figure [ 6 show the expected energy-integrated event rates of $\nu_{e}$

$$
\frac{d N}{d t} \equiv \int_{E_{\mathrm{th}}}^{\infty} \frac{d^{2} N}{d E_{e} d t} d E_{e}
$$

where the integrand in right hand side is the expected event number of neutrinos defined by Eq. (8), and $E_{\text {th }}$ are the threshold energies which are equal to $7 \mathrm{MeV}$ and $5 \mathrm{MeV}$ for SK and SNO detectors, respectively, as explained in section [IC. Left and right panels of Figure 6 are for the cases of normal and inverted hierarchies, respectively. The lower part of 
each panel shows the ratio of the event rates with and without shock. We calculate for the four cases of the mixing angles: $\sin ^{2} 2 \theta_{13}=10^{-2}$ (red), $10^{-3}$ (green), $10^{-4}$ (blue) and $10^{-5}$ (purple).

In the case of normal hierarchy (left panel of Figure 6), the event rate of $\nu_{e}$ of each parameter is not separated clearly from one another. The event rate is commonly about 70 at $t=0 \mathrm{~s}$ and decreases to about 3 at $t=10 \mathrm{~s}$. On the other hand, the ratio of the event rates of $\nu_{e}$ with and without shock shows some different $\theta_{13}$ dependence (left lower panel of Figure 6). After $2 \mathrm{~s}$, we can see slight enhancement of this ratio. We find from this change that the shock front does not reach H-resonance before $\sim 2$ s. Once the shock wave reaches H-resonance region the event rate increases from the low-energy neutrinos because of energy dependence of the resonance density Eq. (6). This ratio then decreases after $3 \mathrm{~s}$ because the event rate of the high-energy neutrinos decreases when the shock wave propagates through the resonance. The obtained time profile thus depends on the $\theta_{13}$ values. The effect of shock propagation appears most clearly in the case of $\sin ^{2} 2 \theta_{13}=10^{-3}$ (green). However, the decrease in the ratio of the event rates is by at most $15 \%$ in the case of small $\theta_{13}$. It is smaller than the case for $\sin ^{2} 2 \theta_{13}=10^{-3}$ at any time for the other $\theta_{13}$ values.

In the case of the inverted mass hierarchy (right panel of Figure 6), the time variation of the event rate of $\nu_{e}$ does not depend on $\theta_{13}$. Shock wave effect is not clearly seen (lower part of right panel of Figure 6). The adiabaticity of $\nu_{e}$ is not influenced by the shock wave because $\nu_{e}$ is not related to H-resonance in the case of inverted hierarchy. Therefore, the spectra of $\nu_{e}$ do not change for any values of $\theta_{13}$.

Figure 7 is the same as Figure 6, but for $\bar{\nu}_{e}$. In the case of the normal hierarchy (left panel of Figure (7), the event rate and the shock wave effect of $\bar{\nu}_{e}$ are insensitive to $\theta_{13}$ throughout the time profile, and event rate decreases from about 3,200 at $t=0 \mathrm{~s}$ to about 100 at $t=10$ s. This is attributed to the fact that the adiabaticity of $\bar{\nu}_{e}$ is not influenced by $\theta_{13}$ or the shock wave as discussed in the previous section.

In the case of the inverted hierarchy (right panel of Figure 7), the behavior of event rate of $\bar{\nu}_{e}$ is different from $\nu_{e}$ in normal hierarchy. At $t=0 \mathrm{~s}$, the event rate is about 4,200 for $\sin ^{2} 2 \theta_{13}=10^{-2}$ and $10^{-3}$, and it is about $3,200-3,500$ for $\sin ^{2} 2 \theta_{13}=10^{-5}-10^{-4}$. The event rate for large $\theta_{13}$ (i.e. $\sin ^{2} 2 \theta_{13}=10^{-2}$ and $10^{-3}$ ) is larger than that for small $\theta_{13}$ (i.e. $\sin ^{2} 2 \theta_{13}=10^{-4}$ and $\left.10^{-5}\right)$. When H-resonance is adiabatic, almost all $\bar{\nu}_{e}$ are completely converted from $\bar{\nu}_{x}$. On the other hand, when H-resonance is non-adiabatic, some $\bar{\nu}_{e}$ remain 
as $\bar{\nu}_{e}$ even after the neutrinos get out of the star. As the result, the fraction of $\bar{\nu}_{e}$ converted from $\bar{\nu}_{x}$ is small. The average energies of the three flavor neutrinos just emitted from the proto-neutron star satisfy the following relation; $\bar{E}_{\nu_{e}}<\bar{E}_{\bar{\nu}_{e}}<\bar{E}_{\nu_{x}}$. Therefore, the average energy of $\bar{\nu}_{e}$ in adiabatic case becomes higher than that in non-adiabatic case. As the main reaction cross section for $\bar{\nu}_{e}$ is proportional to $E_{\nu}^{2}$ as discussed in section IC, the detection probability of high-energy neutrinos is larger than that of low-energy neutrinos. Therefore, the event rate of $\bar{\nu}_{e}$ for large $\theta_{13}$ (adiabatic case) becomes much larger than that for small $\theta_{13}$ (non-adiabatic case). This is the reason for large split between the expected event rates for large $\theta_{13}$ (i.e. $\sin ^{2} 2 \theta_{13}=10^{-2}$ and $10^{-3}$ ) and small $\theta_{13}$ (i.e. $\sin ^{2} 2 \theta_{13}=10^{-4}$ and $10^{-5}$ ). We find difference of event rates by $\sim 1,000$ at $t=0$ between the two cases.

Furthermore, the ratio of the event rates for $\bar{\nu}_{e}$ which exhibits the shock wave propagation effect changes dramatically when $\sin ^{2} 2 \theta_{13}$ is large (right lower panel of Figure 7). The ratio of $\bar{\nu}_{e}$ increases at around $2 \mathrm{~s}$, and decreases after $3 \mathrm{~s}$, which is similar to $\nu_{e}$ event rates in normal hierarchy (left lower panel of Figure 6). The mechanism is quite similar to each other so that the shock wave propagation through the H-resonance starts from low energy neutrinos to high energy neutrinos, which eventually decreases the event rates. However, the decrease in the ratio of the $\bar{\nu}_{e}$ event rates is remarkable by at most $30 \%$ for $\sin ^{2} 2 \theta_{13}=10^{-3}$. On the other hand, the ratio changes weakly for the other $\theta_{13}$ values because H-resonance is non-adiabatic regardless of the shock wave, although its degree is still larger than the $\nu_{e}$ event rate (see left lower panel of Figure 6).

The event rate of $\bar{\nu}_{e}$ is extremely larger than that of $\nu_{e}$ (for a given neutrino oscillation parameter set). This is mainly because the total cross section of $\bar{\nu}_{e}$-induced reactions is about $10^{2}$ times larger than that of $\nu_{e}$. Moreover, the total cross section of $\bar{\nu}_{e}$-induced reactions is almost proportional to the square of neutrino energy, but the total cross section of $\nu_{e^{-}}$ induced reactions is linearly proportional to the neutrino energy. Owing to this different energy dependence of the cross sections, one can expect that the effect of the shock wave should be identified more clearly in the $\bar{\nu}_{e}$ events.

In practice, since the shock effect is folded in the observed event rates of $\nu_{e}$ and $\bar{\nu}_{e}$, we should carry out careful theoretical analysis. The observed event rates are statistically uncertain of the order of their square root. As for $\nu_{e}$ in normal hierarchy, the statistical error is the same order as the change in the ratio $\sim 15 \%$ of the event rates with and without the shock effect because absolute number of expected event rates are small $\frac{d N}{d t} \lesssim 70$ (Figure 
6): Actually, $\frac{d N}{d t} \sim 10$ at $t \sim 6$ s when the shock effect becomes maximum strength, and thereby $\sqrt{\frac{d N}{d t}} / \frac{d N}{d t} \sim 0.3$. On the other hand, for $\bar{\nu}_{e}$ in inverted hierarchy, the statistical error is expected to be small enough because of large absolute number of expected event rates $\frac{d N}{d t} \lesssim 4000$ (Figure 7): In this case, since $\frac{d N}{d t} \sim 500$ at $t \sim 6 \mathrm{~s}, \sqrt{\frac{d N}{d t}} / \frac{d N}{d t} \sim 0.05$ which is much smaller than the maximal change in the ratio $\sim 30 \%$ of the event rates.

\section{Ratio of high- to low-energy neutrino events}

There is a potential difficulty in identifying the influence of the shock wave upon the observed neutrino events because time evolution of the original neutrino spectra, which is unaffected by the shock wave as well as the MSW effect, is unknown. The original neutrino spectra must be assumed theoretically a priori. We therefore look for another useful observable that should show a clear signature of the shock wave effect even when we do not know the time evolution of the original neutrino spectra.

\section{Time-integrated ratio of neutrino events}

We consider now the ratio of the high-energy component to the low-energy component of time-integrated neutrino events. This ratio $R_{\mathrm{x}}$ is defined by [32],

$$
R_{\mathrm{x}} \equiv \frac{\int_{0 \mathrm{~s}}^{10 \mathrm{~s}} \int_{20 \mathrm{MeV}}^{60 \mathrm{MeV}} \frac{d^{2} N}{d E_{e} d t} d E_{e} d t}{\int_{0 \mathrm{~s}}^{10 \mathrm{~s}} \int_{E_{\mathrm{th}}}^{20 \mathrm{MeV}} \frac{d^{2} N}{d E_{e} d t} d E_{e} d t}
$$

where $\mathrm{x}$ refers to $\mathrm{SK}$ or $\mathrm{SNO}$ and $E_{\mathrm{th}}=7 \mathrm{MeV}(\mathrm{SK})$ and $5 \mathrm{MeV}$ (SNO) as explained below Eq. (18). We set here the boundary between high- and low-energy components to be 20 $\mathrm{MeV}$ because the original energy spectra satisfy the conditions $\phi_{\nu_{e}}^{\text {org }}=\phi_{\nu_{x}}^{\text {org }}$ and $\phi_{\bar{\nu}_{e}}^{\text {org }}=\phi_{\nu_{x}}^{\text {org }}$ at $E_{\nu}=18.7 \mathrm{MeV}$ and $23.3 \mathrm{MeV}$, respectively.

Figure 8 shows $R_{\text {SK }}$ vs $R_{\text {SNO }}$ for various mixing angles $\sin ^{2} 2 \theta_{13}=10^{-2}$ (red), $10^{-3}$ (green), $10^{-4}$ (blue) and $10^{-5}$ (purple). Left panel of Figure 8 is in normal hierarchy, and right panel is in inverted hierarchy. Closed and open circles are the calculated results with and without shock wave effect, respectively. Note that the scale of each panel is different.

In the inverted hierarchy, we see large variations of the ratios; $R_{\mathrm{SK}} \sim 1.8-4.6$, and $R_{\mathrm{SNO}} \sim 2.3-4.3$. In addition, $R_{\mathrm{SK}}$ and $R_{\mathrm{SNO}}$ show a clear correlation because the most 
dominated reactions for SK and SNO detectors are the $\bar{\nu}_{e}$-induced charged current reactions; $\bar{\nu}_{e}+p \rightarrow e^{+}+n$ for SK and $\bar{\nu}_{e}+d \rightarrow n+n+e^{+}$for SNO. Both ratios $R_{\mathrm{SK}}$ and $R_{\mathrm{SNO}}$ increases with the mixing angle $\theta_{13}$. For a given $\theta_{13}$, the ratios in the case with shock are smaller than those without the shock. On the other hand, in the normal hierarchy, the ratio shows a small dependence on $\sin ^{2} 2 \theta_{13}$ for $R_{\mathrm{SK}}$ and $R_{\mathrm{SNO}}$. In practice, we see only small variation of the ratios; $R_{\mathrm{SK}} \sim 1.76-1.8$, and $R_{\mathrm{SNO}} \sim 2.2-2.5$. These characteristics in the parameter dependence of $R_{\mathrm{SK}}$ and $R_{\mathrm{SNO}}$ are consistent with the result of ref. [32].

The parameter dependence which we confirmed in $R_{\mathrm{SK}}$ and $R_{\mathrm{SNO}}$ as discussed above could be an important observational signature to discriminate neutrino mass hierarchy, normal or inverted, as well as the mixing angle, $\theta_{13}$. In normal hierarchy, the ratios $R_{\mathrm{SK}}$ and $R_{\mathrm{SNO}}$ are small regardless of the mixing angle $\theta_{13}$ and the shock effect. Furthermore, $R_{\mathrm{SNO}}$ changes a little according to the value of $\theta_{13}$ and $R_{\mathrm{SK}}$ hardly changes. We defined $R_{\mathrm{x}}$ as the total events of all flavors. Most of the events detected in SK is $\bar{\nu}_{e}$. As a result, the variation of $\nu_{e}$ events is hindered by larger $\bar{\nu}_{e}$ events unchanged by the $\theta_{13}$ variation. On the other hand, the events of SNO contain the events of $\nu_{e}$ as much as that of $\bar{\nu}_{e}$, because the cross section of $\nu_{e}$-induced reaction is of the same order as that of $\bar{\nu}_{e}$-induced reaction. Therefore, we can see the variation of $\nu_{e}$. However, the variation of $\nu_{e}$ is small because the number of events is not so different between $\nu_{e}$ and $\bar{\nu}_{e}$. In inverted hierarchy, the ratios $R_{\mathrm{SK}}$ and $R_{\mathrm{SNO}}$ correlate with the mixing angle $\theta_{13}$ and the shock effect. Larger $\theta_{13}$ value indicates larger $R_{\mathrm{SK}}$ and $R_{\mathrm{SNO}}$ ratios. However, the shock effect reduces $R_{\mathrm{SK}}$ and $R_{\mathrm{SNO}}$ even in large $\theta_{13}$ value. Therefore, we do not distinguish the value of $\theta_{13}$ and the shock effect in $R_{\mathrm{SK}}$ and $R_{\mathrm{SNO}}$.

\section{Time-dependent ratio of neutrino events}

In the previous subsection, we discussed the ratio of high-energy to low-energy events, $R_{\mathrm{x}}$, which is the ratio of the neutrino events integrated over the time. We also evaluated in Sec. IIIC the time-dependent event rates integrated over the neutrino energy. Figures 6 and 7 indicate that some effects of shock wave propagation could be observed at later times when the shock wave reaches H-resonance. However, we apparently lose some important information on the effects of shock wave in either case. In this subsection, we explore for the signature of the shock wave effects by taking account of double differential as to both 
time and energy.

We here define time-dependent ratio of the events of high-energy to low-energy neutrinos,

$$
R_{\mathrm{x}}(t) \equiv \frac{\int_{20 \mathrm{MeV}}^{60 \mathrm{MeV}} \frac{d^{2} N}{d E_{e} d t} d E_{e}}{\int_{E_{\mathrm{th}}}^{20 \mathrm{MeV}} \frac{d^{2} N}{d E_{e} d t} d E_{e}},
$$

where $\mathrm{x}$ refers to $\mathrm{SK}$ or SNO. Figure 9 shows this ratio $R_{\mathrm{x}}(t)$ as a function of time. The values of $\sin ^{2} 2 \theta_{13}$ are $10^{-2}, 10^{-3}, 10^{-4}$ and $10^{-5}$ from top to bottom panels. Left panels are for normal hierarchy and right panels are for inverted hierarchy. Solid and dashed lines are the calculated results with and without shock wave, respectively. Note that the scale of each panel is different.

First, we show $R_{\mathrm{SNO}}(t)$ in normal hierarchy in left panels of Figure 9. The effect of shock wave is seen, but it is a very small effect of at most about $10 \%$. As for observed $\nu_{e}$ spectrum in normal hierarchy, $70 \%$ is the original $\nu_{x}$ and $30 \%$ is the original $\nu_{e}$ in the non-adiabatic case, though almost $100 \%$ is the original $\nu_{x}$ in the adiabatic case. On the other hand, $70 \%$ of the observed $\nu_{e}$ is the original $\bar{\nu}_{e}$ and $30 \%$ is the original $\nu_{x}$ in inverted hierarchy. Moreover, the shock effect of $\nu_{e}$ in normal hierarchy is washed out by neutral current and charged current of $\bar{\nu}_{e}$ because the target of neutrino detection is deuteron. Therefore, the variation of the event rate ratio is small in the normal hierarchy. There is, however, possibility of finding shock wave effect if much larger events are detected by the next generation detector.

Second, we show $R_{\mathrm{SK}}(t)$ in inverted hierarchy in right panels of Figure 9. The event rate ratio $R_{\mathrm{SK}}(t)$ is constant in early times and strongly depends on $\sin ^{2} 2 \theta_{13}$. It is 4.7 and 1.8 in the case of $\sin ^{2} 2 \theta_{13}=10^{-3}$ and $10^{-5}$, respectively, at $t=0 \mathrm{~s}$. The value of $R_{\mathrm{SK}}(t)$ is large when $\sin ^{2} 2 \theta_{13}$ is large, because the H-resonance is adiabatic and the average energy of $\bar{\nu}_{e}$ is high. The small $R_{\mathrm{SK}}(t)$ value is due to non-adiabatic state of H-resonance.

When the shock wave effects are included and $\sin ^{2} 2 \theta_{13}$ is $10^{-3}$ (large), $R_{\mathrm{SK}}(t)$ greatly changes with time. Although $R_{\mathrm{SK}}(t)$ is 4.7 until $2 \mathrm{~s}$, it decreases to $\sim 2$, which is closer to the ratio in the case of non-adiabatic state in $4-8 \mathrm{~s}$. This decrease is due to the change of the adiabaticity of H-resonance according to the shock propagation. The H-resonance is adiabatic before the shock arrival to the resonance. In contrast, the H-resonance is non-adiabatic while the shock wave propagates in the resonance, and $R_{\mathrm{SK}}(t)$ approaches $R_{\mathrm{SK}}(t) \sim 1.8$ which is the ratio in small $\sin ^{2} 2 \theta_{13}$ case (see the bottom panel). 
When $\sin ^{2} 2 \theta_{13}$ is as small as $10^{-5}$, we see only small differences of $R_{\mathrm{SK}}(t)$ between the cases with and without the shock wave and hardly see the time dependence. The H-resonance is non-adiabatic whenever the shock wave is on the resonance or not in this case of small $\sin ^{2} 2 \theta_{13}$.

To conclude, we can see a clear signature of the shock wave in the time-dependent highenergy to low-energy ratio of $R_{\mathrm{x}}(t)$ although the influence of the shock wave is less clearly seen in the ratio of time integrated event rates, $R_{\mathrm{x}}$. Therefore, the observations of the time evolution of $R_{\mathrm{x}}(t)$ is quite an important observable to constrain the neutrino oscillation parameters and to find the effects of shock propagation in supernovae.

\section{E. Further discussion}

Fogli et al.[27] found qualitatively similar result to ours in the calculated event rates with and without forward shock in the case of inverted hierarchy for $\sin ^{2} 2 \theta_{13}=10^{-2}$. Their results are however different from ours quantitatively for several reasons. For one, their event rates at the neutrino energy $45 \pm 5 \mathrm{MeV}$ are calculated by assuming the next generation detector of 0.4 Mton pure water that is 12.5 bigger than 32 kton for SK. We can scale our calculated results by 12.5 times in order to remove this apparent difference. The second reason is that they adopted completely different supernova model from ours. Although it is hard to reconcile the difference between the two models, we tried to compare at least the effect of shock wave propagation in the following manner.

We should, first, refer to our result of the event numbers at the same neutrino energy 45 $\pm 5 \mathrm{MeV}$ at $5 \mathrm{~s}$ after the core bounce for $\sin ^{2} 2 \theta_{13}=10^{-3}$. This aims to compare the two results of the event rates so that the shock wave effect appears most remarkably in both calculations in the case of inverted hierarchy. Second, we should remove the bias based on different supernova models especially arising from different density profiles from each other. For this purpose we normalize our event number without shock to Fogli's event number without shock. We thus obtain finally the following event numbers; 135 with shock and 300 without shock in inverted hierarchy. These scaled and normalized numbers should be compared with Fogli's results; 130 with shock and 300 without shock. Note again that the latter number in either calculation is the same by definition of normalization. Our numerical calculations show $55.0 \%$ decrease in the event number as a resultant net effect of 
shock wave propagation, which is in reasonable agreement with $56.7 \%$ decrease as shown by their calculations. We thus conclude that their theoretical calculations and ours of the neutrino event rates agree with each other despite several essential differences: We solved in Eq. (1) both flavor conversion of neutrinos and supernova density profile numerically in our method described in Sec. 1, while Fogli et al. [27] applied an artificial model of density profile of shock wave propagation without solving dynamical supernova explosion.

For further comparison, let us analytically estimate the decrease in the neutrino events in the non-adiabatic case (with shock) compared with the adiabatic case (without shock). In the non-adiabatic case, we define the survival probability of $\bar{\nu}_{e}$ as $\bar{P}=0.7$ in our model, and we use the result of $\bar{\nu}_{e}$ in the case of normal hierarchy in Fogli's model. We set $\bar{P}=0$ in the adiabatic case. Thus estimated analytical results show $62.3 \%$ and $63.0 \%$ decrease in the neutrino events from adiabatic to non-adiabatic cases in our model and their model, respectively. These values are close enough to each other, and $\sim 60 \%$ are not very different from $\sim 55.0 \%$ which was inferred from numerical calculations as discussed in the previous paragraph. This fact justifies that our treatment of analytical estimates are reasonable. Therefore, regardless of all possible differences between the two models, the supernova neutrinos provide a powerful tool to indicate the shock wave propagation inside supernova if $\sin ^{2} 2 \theta_{13}$ is large in the inverted hierarchy.

We assumed that $\mathrm{CP}$ violating phase is zero and $\sin ^{2} 2 \theta_{23}=1$ throughout this study. However, CP-phase is unknown and there is an uncertainty in the mixing angle $\theta_{23}$. Here we discuss the dependence of the shock effect on neutrino flavor transitions on CP-phase and $\theta_{23}$. Transition probabilities of neutrinos in arbitrary density profile have been studied theoretically in [65], where it was found that the transition probabilities of $\nu_{e} \rightarrow \nu_{e}$ and $\bar{\nu}_{e} \rightarrow \bar{\nu}_{e}$ do not depend on CP-phase and mixing angle $\theta_{23}$ at all. Furthermore, one can show from their formula in [65] that the sum of the transition probabilities of $\nu_{\mu} \rightarrow \nu_{e}$ and $\nu_{\tau} \rightarrow \nu_{e}\left(\bar{\nu}_{\mu} \rightarrow \bar{\nu}_{e}\right.$ and $\left.\bar{\nu}_{\tau} \rightarrow \bar{\nu}_{e}\right)$ are totally free from CP-phase and $\theta_{23}$. Since the spectra of $\nu_{\mu}$ and $\nu_{\tau}\left(\bar{\nu}_{\mu}\right.$ and $\left.\bar{\nu}_{\tau}\right)$ are the same at the neutrino sphere, the transition probability from $\nu_{\mu}$ or $\nu_{\tau}\left(\bar{\nu}_{\mu}\right.$ or $\left.\bar{\nu}_{\tau}\right)$ to $\nu_{e}\left(\bar{\nu}_{e}\right)$ can be written as a half of the sum of the transition probabilities of $\nu_{\mu} \rightarrow \nu_{e}$ and $\nu_{\tau} \rightarrow \nu_{e}\left(\bar{\nu}_{\mu} \rightarrow \bar{\nu}_{e}\right.$ and $\left.\bar{\nu}_{\tau} \rightarrow \bar{\nu}_{e}\right)$. The transition probability from $\nu_{\mu}$ or $\nu_{\tau}$ $\left(\bar{\nu}_{\mu}\right.$ or $\left.\bar{\nu}_{\tau}\right)$ to $\nu_{e}\left(\bar{\nu}_{e}\right)$ does not depend on CP-phase and $\theta_{23}$. We thus conclude that there is no effect of CP-phase and mixing angle $\theta_{23}$ on the supernova neutrino spectra.

If the influence of the shock wave is seen very early $(t \leq 1 s)$ in the observation of the 
supernova neutrinos, it might mean that the shock wave reaches H-resonance $\left(\sim 1000 \mathrm{~g} / \mathrm{cm}^{3}\right)$ very early after core bounce. Then, we would expect that this kind of supernova explosion exhibits a strong shock wave effect in the direction of observer. Moreover, assume that we could know viewing angle from the axis of a supernova in optical and radio observations. If the viewing angle is small and the influence of the shock wave is seen very early, this supernova has strong shock like a narrow beaming jet along the axis of supernova explosion. Therefore, we would expect that this supernova explodes in the mechanism associated with the jet formation [66 72]. If the viewing angle is large and the influence of the shock is seen very early, we would expect that this supernova has a rather wide jet and may be close to the spherical symmetric explosion.

On the other hand, if the influence of the shock wave is seen in the supernova neutrinos at relatively later time, and the viewing angle is small, we would expect that this explosion is also close to the spherical symmetry without jet. Therefore, we would expect that this supernova explodes in the mechanism without a jet. Details of the influence of neutrino oscillation on jet explosion are discussed in [73].

Recently, oxygen emission-line profiles from supernovae are observed, which could be a signature of an aspheric explosion [74]. We would study the asphericity of the supernova explosions more in detail from such an optical observation and simultaneous detection of the supernova neutrinos.

The flavor-exchange effects by the neutrino self-interactions might also be important because of their huge flux immediately after the emission out of the proto-neutron star (e.g., [75, 76]). This could change initial neutrino spectrum from what we assumed here.

\section{CONCLUSION}

We investigated the neutrino signal to study the effect of the shock wave propagation as well as the dependence on unknown neutrino oscillation parameters of mass hierarchy and $\theta_{13}$ by carrying out numerical calculations of the neutrino event number using the simulation result of a supernova explosion. We followed adiabatic collapse of iron core, core bounce, and the shock wave propagation in the stellar envelope for a long time ( $\gtrsim 10 \mathrm{~s}$ ) using general relativistic hydrodynamical code and realistic density profile. Then, we could calculate detailed time evolution of the event number rate and spectra of the supernova neutrinos. 
The expected event rate of $\bar{\nu}_{e}$ in the case of the inverted mass hierarchy and that of $\nu_{e}$ in the normal mass hierarchy depend on the mixing angle $\theta_{13}$. When $\sin ^{2} 2 \theta_{13}$ is larger than $\sim 10^{-4}$, the influence of the shock wave appears in the observation after $2 \mathrm{~s}$ in our model. It is the time when the shock wave reaches the H-resonance. Therefore, the shock effect and the constraint on $\theta_{13}$ can be inferred even by the event rate integrated by the whole energy range. However, it is difficult to distinguish the influence of the shock wave and the neutrino oscillation parameters only from the time evolution of the event rate.

The time-integrated ratio of the events of high-energy to low-energy neutrinos is another indicator to examine the shock effect and constrain mass hierarchy and $\theta_{13}$. The parameter dependence of the time integrated ratio $R_{\mathrm{x}}(\mathrm{x}=\mathrm{SK}$ or SNO) could be an important observational signature to discriminate neutrino mass hierarchy, normal or inverted, as well as the mixing angle, $\theta_{13}$. Both of the ratios $R_{\mathrm{SK}}$ and $R_{\mathrm{SNO}}$ correlate with the value of $\theta_{13}$ in inverted mass hierarchy. Therefore, the neutrino oscillation parameters $\theta_{13}$ and the mass hierarchy, might be able to be constrained by this ratio. The shock effect reduces the ratios $R_{\mathrm{SK}}$ and $R_{\mathrm{SNO}}$. This effect is not distinguished with smallness of $\theta_{13}$.

The time-dependent ratio of high- to low-energy neutrino events is more preferable indicator to find out the shock effect clearly. The ratio decreases after $2 \mathrm{~s}$ for $R_{\mathrm{SNO}}(t)$ in normal hierarchy and for $R_{\mathrm{SK}}(t)$ in inverted hierarchy. The decrease is the most remarkable in the case of $\sin ^{2} 2 \theta_{13}=10^{-3}$ and more clearly in inverted hierarchy. The dramatic decrease in the ratio could be a clear signal for the shock wave effect and would constrain the minimum value of $\sin ^{2} 2 \theta_{13}$. Therefore, observations of the time-dependent ratio of the high- to lowenergy neutrino event are an important observable to find the effects of shock propagation in supernovae.

\section{Acknowledgments}

We would like to thank K. Kotake for fruitful discussions. SK thanks to T. Takiwaki and S. Harikae for help comments on the numerical scheme. This research work was supported 
in part by Research Fellowships of the Japan Society for the Promotion of Science (JSPS).

[1] K. Hirata, Y. Kajita, M. Koshiba, M. Nakahata, and Y. Oyama, Phys. Rev. Lett., 58, 1490 (1987).

[2] R. M. Bionta, G. Blewitt, C. B. Bratton, D. Caspere, and A. Ciocio, Phys. Rev. Lett., 58, 1494 (1987).

[3] K. Sato and H. Suzuki, Phys. Rev. Lett., 58, 2722 (1987).

[4] H. Suzuki and K. Sato, Prog. Theor. Phys., 79, 725 (1988).

[5] H. Suzuki and K. Sato, PASJ, 39, 512 (1987).

[6] H. Suzuki, Supernova neutrinos in Physics and Astrophysics of Neutrino, editted by M. Fukugita and A. Suzuki, Springer-Verlag, Tokyo, 763 (1994).

[7] Y. Totsuka, Rept. Prog. Phys., 55, 377 (1992).

[8] KamLAND Collaboration, A. Suzuki et al., Present Status of KamLAND, Nucl. Phys., 77 (Proc. Suppl.) 171, (1999).

[9] SNO Collaboration, Phys. Rev. Lett., 87, 071301 (2001).

[10] C. Kraus, and The SNO+ Collaboration, Progress in Particle and Nuclear Physics, 57, 150 (2006).

[11] T. Totani, K. Sato, H. E. Dalged, and J. R. Wilson, ApJ, 496, 216 (1998).

[12] S. Ando, J. F. Beacom, and H. Yüksel, Phy. Rev. Lett., 95, 171101 (2005) astro-ph/0503321.

[13] L. Mosca, Nucl. Phys. B Proc. Suppl., 138, 203 (2005)

[14] K. Nakamura, Int. J. of Mod. Phys. A, 18, 4053 (2003)

[15] Y. Suzuki et al. [TITAND Working Group], hep-ex/0110005.

[16] C. K. Jung, American Institute of Phys. Conf. Ser., 533, 29 (2000) hep-ex/0005046

[17] S. Fukuda, Y. Fukuda, M. Ishitsuka, Y. Itow, T. Kajita, J. Kameda, K. Kaneyuki, K. Kobayashi, Y. Koshio, M. Miura, and 108 coauthors, Phys. Rev. Lett., 86, 5656 (2001) hep-ex/0103033.

[18] Q. R. Ahmad, R. C. Allen, T. C. Andersen, J. D. Anglin, J. C. Barton, E. W. Beier, M. Bercovitch, J. Bigu, S. D. Biller, and 169 coauthors, Phys. Rev. Lett., 89, 1302 (2002).

[19] M. Maltoni, T. Schwetz, M. A. Tortola, and J. W. F. Valle, New J. Phys., 06, 122 (2004) 
hep-ph/0405172.

[20] CHOOZ Collaboration, M. Apollonio et al., Phys. Lett. B, 466, 415 (1999) hep-ex/9907037.

[21] K. Takahashi, M. Watanabe, K. Sato and T. Totani, Phys. Rev. D, 64, 093004 (2001) hep-ph/0105204.

[22] C. Lunardini and A. Yu. Smirnov JCAP, 0306, 009 (2003) hep-ph/0302033.

[23] G. Fogli, E. Lisi, D. Montanino and A. Palazzo, Phys. Rev. D, 65, 073008 (2002).

[24] A. S. Dighe and A. Yu. Smirnov, Phys. Rev. D, 62, 3007 (2000) hep-ph/9907423.

[25] A. S. Dighe, M. T. Keil and G. G. Raffelt, JCAP, 0306, 005 (2003) hep-ph/0303210.

[26] S. Kawagoe, T. Kajino, H. Suzuki, K. Sumiyoshi, and S. Yamada, J. Phys. Conf. Ser., 120, 052020 (2008).

[27] G. Fogli, E. Lisi, A. Mirizzi, and D. Montanino, JCAP, 04, 002 (2005) hep-ph/0412046.

[28] G. Fogli, E. Lisi, A. Mirizzi, and D. Montanino, Phys. Rev. D, 68, 033005 (2003) hep-ph/0304056.

[29] K. Nakazato, K. Sumiyoshi, H. Suzuki, and S. Yamada, Phy. Rev. D, 78, 083014 (2008) arXiv:0810.3734

[30] C. Lunardini, B. Müller and H. Th. Janka, Phys. Rev. D, 78, 023016 (2008) arXiv:0712.3000.

[31] A. B. Balantekin, J. M. Fetter, and F. N. Loreti, Phys. Rev. D, 54, 3941 (1996) astro-ph/9604061.

[32] K. Takahashi and K. Sato, PTP, 109, 919 (2003) hep-ph/0205070.

[33] K. Takahashi, K. Sato, A. Burrows, and T. A. Thompson, Phys. Rev. D, 68, 113009 (2003) hep-ph/0306056.

[34] K. Takahashi and K. Sato, Phys. Rev. D, 66, 033006 (2002) hep-ph/0110105.

[35] K. Takahashi, M. Watanabe, and K. Sato, Phys. Lett. B, 510, 189 (2001) hep-ph/0012354.

[36] A. S. Dighe, M. T. Keil, and G. G. Raffelt, JCAP, 0306, 006 (2003) hep-ph/0304150.

[37] C. Lunardini and A. Y. Smirnov, Nucl. Phys. B, 616, 307 (2001) hep-ph/0106149.

[38] S. Ando and K. Sato, Phys. Rev. D, 67, 023004 (2003) hep-ph/0211053.

[39] S. Ando and K. Sato, Phys. Rev. D, 68, 023003 (2003) hep-ph/0305052.

[40] S. Ando and K. Sato, JCAP, 0310, 001 (2003) hep-ph/0309060.

[41] E. K. Akhmedov and T. Fukuyama, JCAP, 0312, 007 (2003) hep-ph/0310119.

[42] A. Ahriche and J. Mimouni, JCAP, 0311, 004 (2003) astro-ph/0306433. 
[43] R. C. Schirato and G. M. Fuller astro-ph/0205390.

[44] S. Kawagoe, T. Kajino, H. Suzuki, K. Sumiyoshi, and S. Yamada, J. Phys. Conf. Ser., 39, $294(2006)$.

[45] K. Takahashi, K. Sato, H. E. Dalhed, and J. R. Wilson, Astropart. Phys., 20, 189, astro-ph/0212195.

[46] R. Tomàs, M. Kachelrieß, G. Raffelt, A. Dighe, H. Th. Janka, and L. Scheck, JCAP 09, 015 (2004) astro-ph/0407132.

[47] S. Yamada, ApJ, 475, 720 (1997) astro-ph/9601042.

[48] H. Shen, H. Toki, K. Oyamatsu, and K. Sumiyoshi, Nucl. Phys. A, 637, 435 (1998) nucl-th/9805035.

[49] F. X. Timmes, and D. Arnett, D., 1999, ApJS, 125, 277 (1999).

[50] S. E. Woosley, and T. Weaver, ApJS, 101, 181 (1995).

[51] K. Sumiyoshi, H. Suzuki, S. Yamada, and H. Toki, Nucl. Phys. A, 730, 227 (2004) nucl-th/0310046.

[52] S. Kawagoe, H. Suzuki, K. Sumiyoshi, and S. Yamada, Proceedings of the Yamada Conference LIX on Inflating Horizons of Particle Astrophysics and Cosmology (H.Suzuki, J.Yokoyama, Y.Suto, and K. Sato, Japan, 2006, pp 277 - 278)

[53] S. Kawagoe, doctor thesis, The graduate university for advanced studies (2008).

[54] M. T. Keil, G. G. Raffelt, and H. -T. Janka, ApJ, 590, 971 (2003) astro-ph/0208035.

[55] T. Yoshida, T. Kajino, and D. H. Hartmann, Phys. Rev. Let., 94, 231101 (2005) astro-ph/0505043.

[56] T. Yoshida, T. Suzuki, S. Chiba, T. Kajino, H. Yokomakura, K. Kimura, A. Takamura, and D. H. Hartmann, ApJ, 686, 448 (2008) [arXiv:0807.2723].

[57] S. E. Woosley, J. R. Wilson, G. J. Mathews, R. D. Hoffman, and B. S. Meyer, ApJ, 433, 229 (1994).

[58] T. Yoshida, M. Terasawa, T. Kajino, and K. Sumiyoshi, ApJ, 600, 204 (2004) astro-ph/0305555.

[59] M. Fukugita and T. Yanagida, Physics of Neutrinos and Applications to Astrophysics, Springer, Berlin (2003).

[60] K. Takahashi, M. Watanabe, and K. Sato, Phys. Rev. B, 510, 189 (2001) hep-ph/0012354.

[61] Y. Fukuda, T. Hayakawa, E. Ichihara, K. Inoue, K. Ishihara, H. Ishino, Y. Itow, T. Kajita, J. 
Kameda, S. Kasuga and 116 coauthors, Phys. Rev. Lett. , 81, 1158 (1998).

[62] J. N. Bahcall, P. I. Krastev, and A. Yu. Smirnov Phys. Rev. B, 477, 401 (2000) hep-ph/9911248.

[63] S. Ying, W. C. Haxton and E. M. Henley, Phys. Rev. D, 40, 3211 (1989).

[64] S. Nakamura, T. Sato, V. Gudkov and K. Kubodera, Phys. Rev. C, 63, 4617 (2001).

[65] H. Yokomakura, K. Kimura and A. Takamura, Phys. Lett. B, 544, 286 (2002).

[66] M. Obergaulinger, M. A. Aloy and E. Muller, Astron. Astrophys., 450, 1107 (2006) astro-ph/0510184.

[67] D. A. Uzdensky and A. I. MacFadyen, Phys. Plasmas 14, 056506 (2007) Phys. Plasmas., 14, 056506 (2007) [arXiv:0707.0576].

[68] S. G. Moiseenko, G. S. Bisnovatyi-Kogan and N. V. Ardeljan, astro-ph/0603789.

[69] A. Burrows, L. Dessart, E. Livne, C. D. Ott and J. Murphy, astro-ph/0702539.

[70] H. Sawai, K. Kotake and S. Yamada, ApJ, 672, 465 (2008) arXiv:0709.1795.

[71] H. Mikami, Y. Sato, T. Matsumoto and T. Hanawa, ApJ, 683, 357 (2008) arXiv:0804.3700.

[72] T. Takiwaki, K. Kotake and K. Sato, ApJ, 691, 1360 (2009) [arXiv:0712.1949.

[73] S. Kawagoe, T. Takiwaki and K. Kotake, accepted for JCAP (2009), [arXiv:0906.3180].

[74] K. Maeda, K. Kawabata, P. A. Mazzali, M. Tanaka, S. Valenti, K. Nomoto, T. Hattori, J. Deng, E. Pian, S. Taubenberger, M. Iye, T. Matheson, A. V. Filippenko, K. Aoki, G. Kosugi, Y. Ohyama, T. Sasaki, and T. Takata, Science, 319, 1220 (2008), [arXiv:0801.1100].

[75] G. Fogli, E. Lisi, A. Marrone, and A. Mirizzi, JCAP, 12, 010 (2007), arXiv:0707.1998.

[76] H. Duan, and J. P. Kneller [arXiv:0904.0974] 


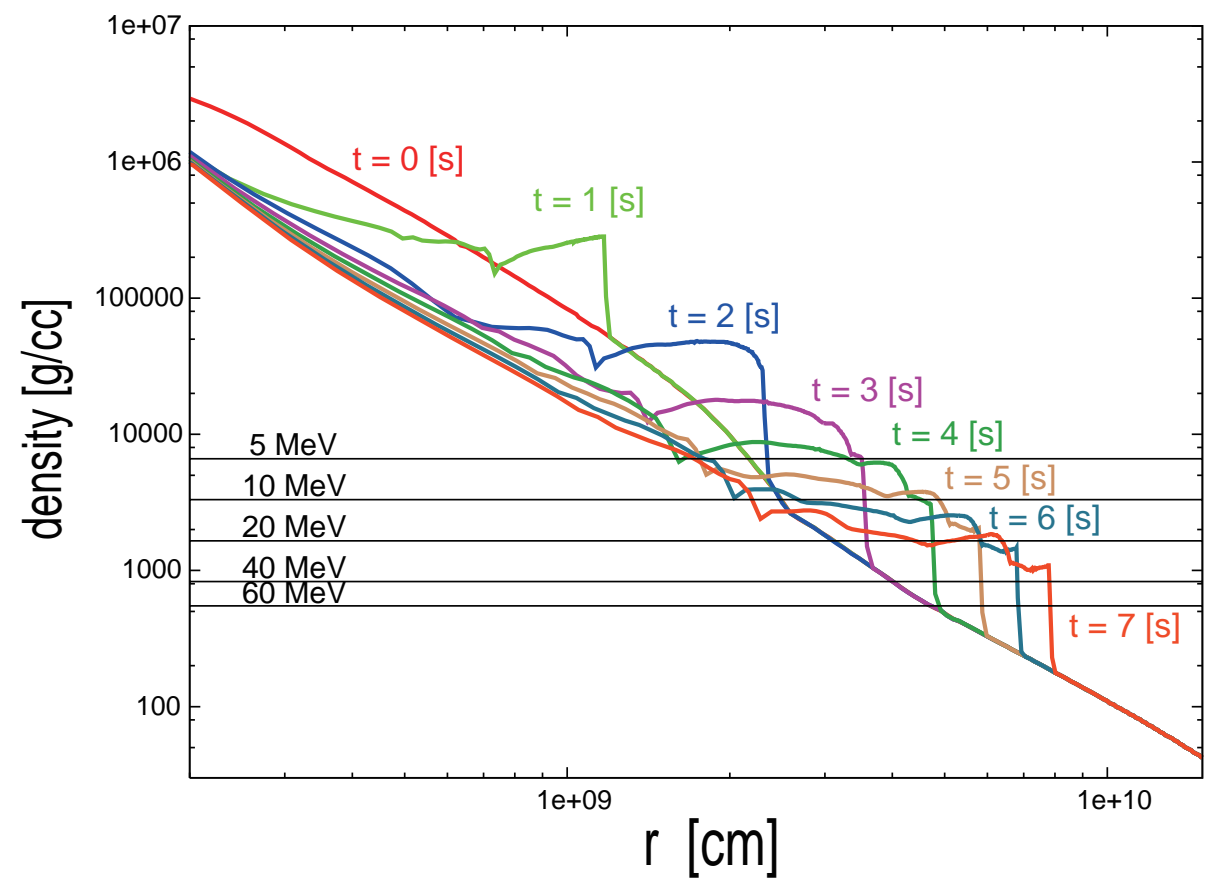

FIG. 1: The density profiles as a function of radius for every 1 second from 0 to 7 seconds after the core bounce. The horizontal lines show the density at the H-resonance point of each neutrino energy. 

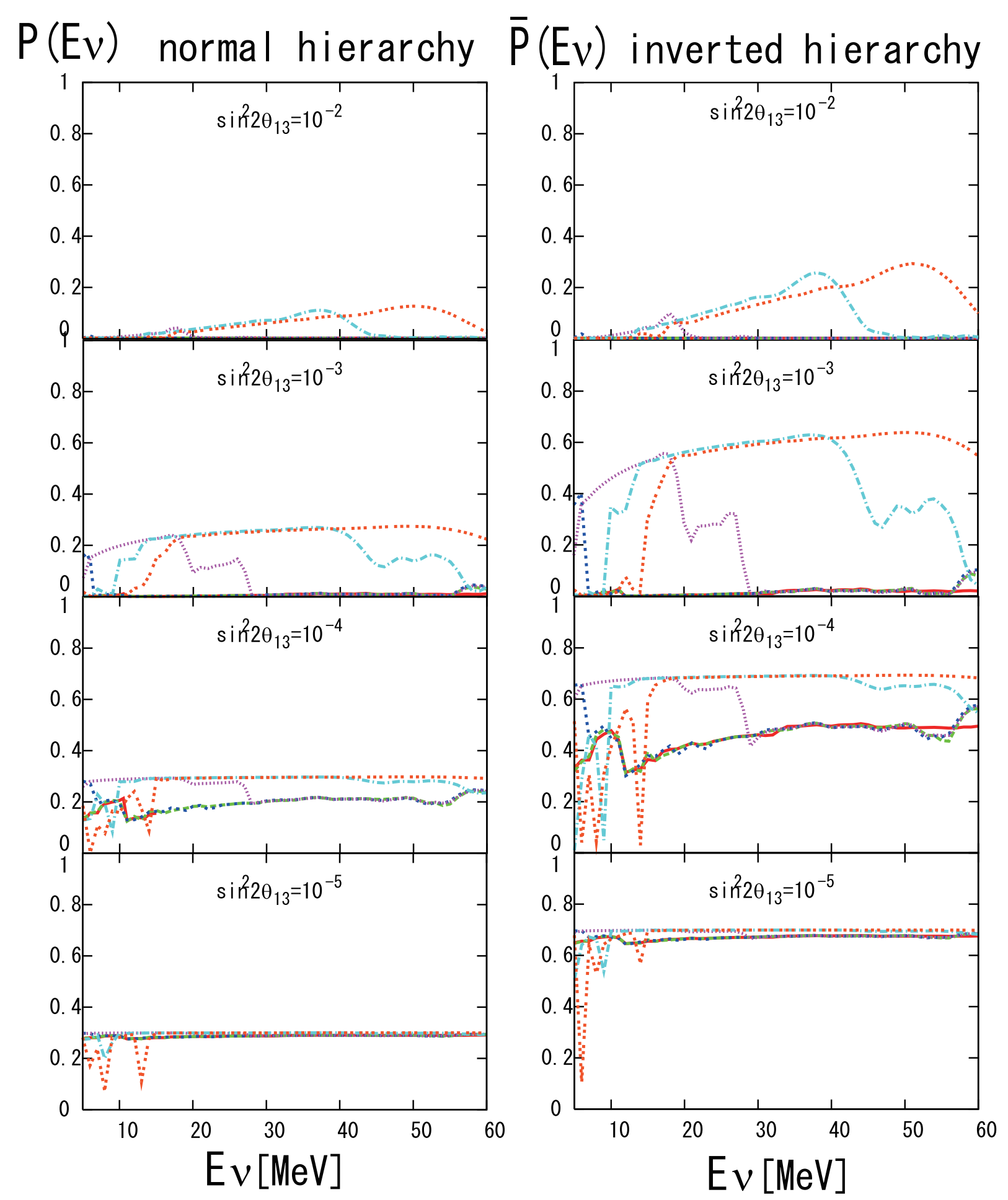

FIG. 2: Calculated results of the survival probabilities, $P\left(E_{\nu}\right)$ and $\bar{P}\left(E_{\nu}\right)$, in every 1 second. Left panels are the survival probabilities of $\nu_{e}, P\left(E_{\nu}\right)$, in normal hierarchy, and right panels are the survival probabilities of $\bar{\nu}_{e}, \bar{P}\left(E_{\nu}\right)$, in inverted hierarchy. Red, green, blue, purple, sky blue and orange lines are $\mathrm{t}=0,1,2,3,4$ and 5 sec after core bounce. The value of $\sin ^{2} 2 \theta_{13}$ is $10^{-2}, 10^{-3}$, $10^{-4}$ and $10^{-5}$ from top to bottom. 

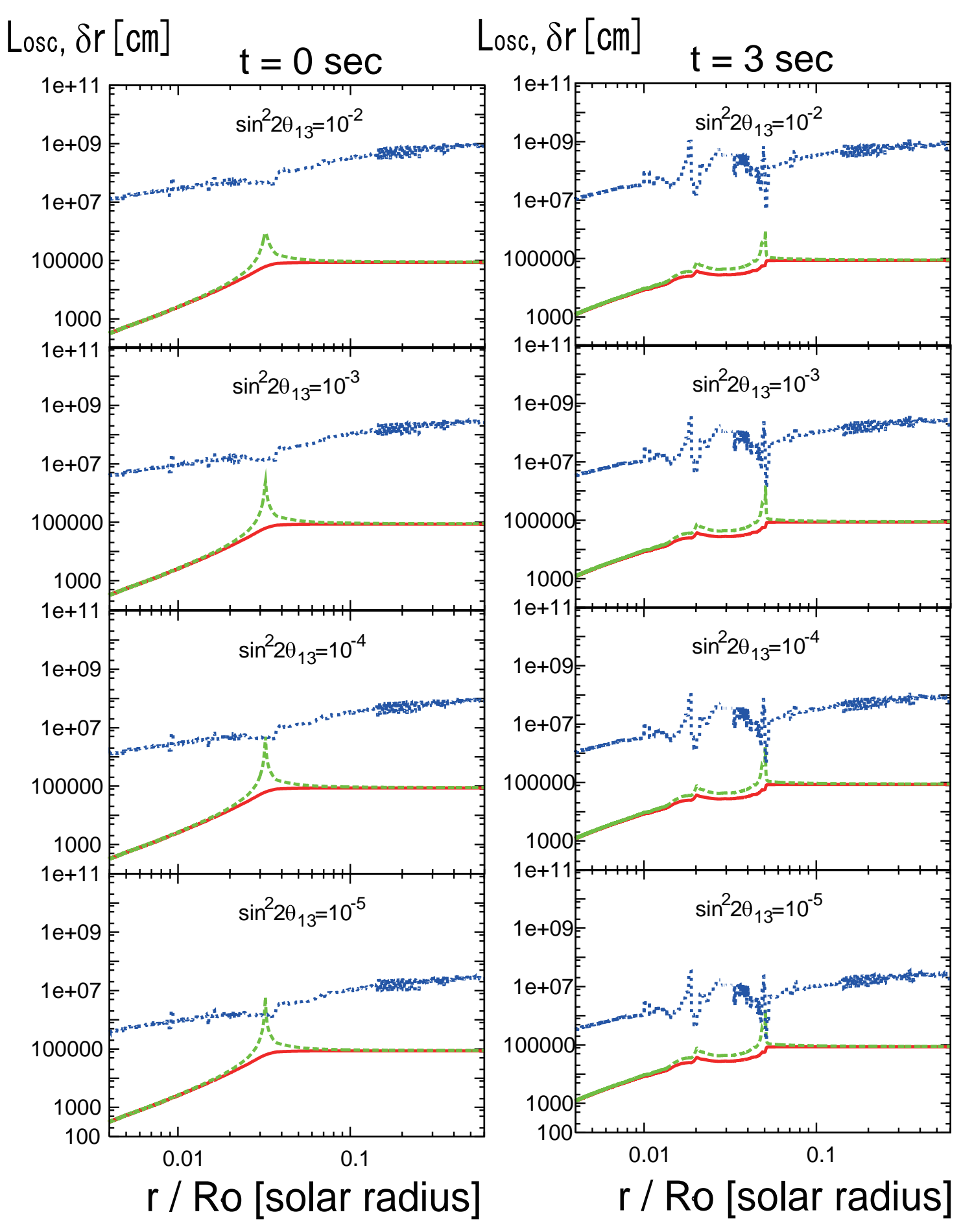

FIG. 3: The oscillation length, $L_{\mathrm{osc}}=\frac{2 E}{\Delta \tilde{m}^{2} \sin 2 \tilde{\theta}}$, and the level crossing length, $\delta r=\frac{\sin 2 \theta}{\cos 2 \theta}\left|\frac{1}{n_{e}} \frac{d n_{e}}{d r}\right|^{-1}$, of neutrinos for $5 \mathrm{MeV}$ in the case of inverted mass hierarchy as a function of a radius $r$ in units of solar radius $R_{\odot}=6.96 \times 10^{10} \mathrm{~cm}$. The left panels are at $t=0 \mathrm{sec}$, and the right panels are at $t$ $=3 \mathrm{sec}$, respectively. We calculate by the value of $\sin ^{2} 2 \theta_{13}=10^{-2}, 10^{-3}, 10^{-4}$ and $10^{-5}$ from top to bottom. Red and green lines are the oscillation length for 13 mass eigenstate for neutrinos and anti-neutrinos, and blue line are the level crossing length. 

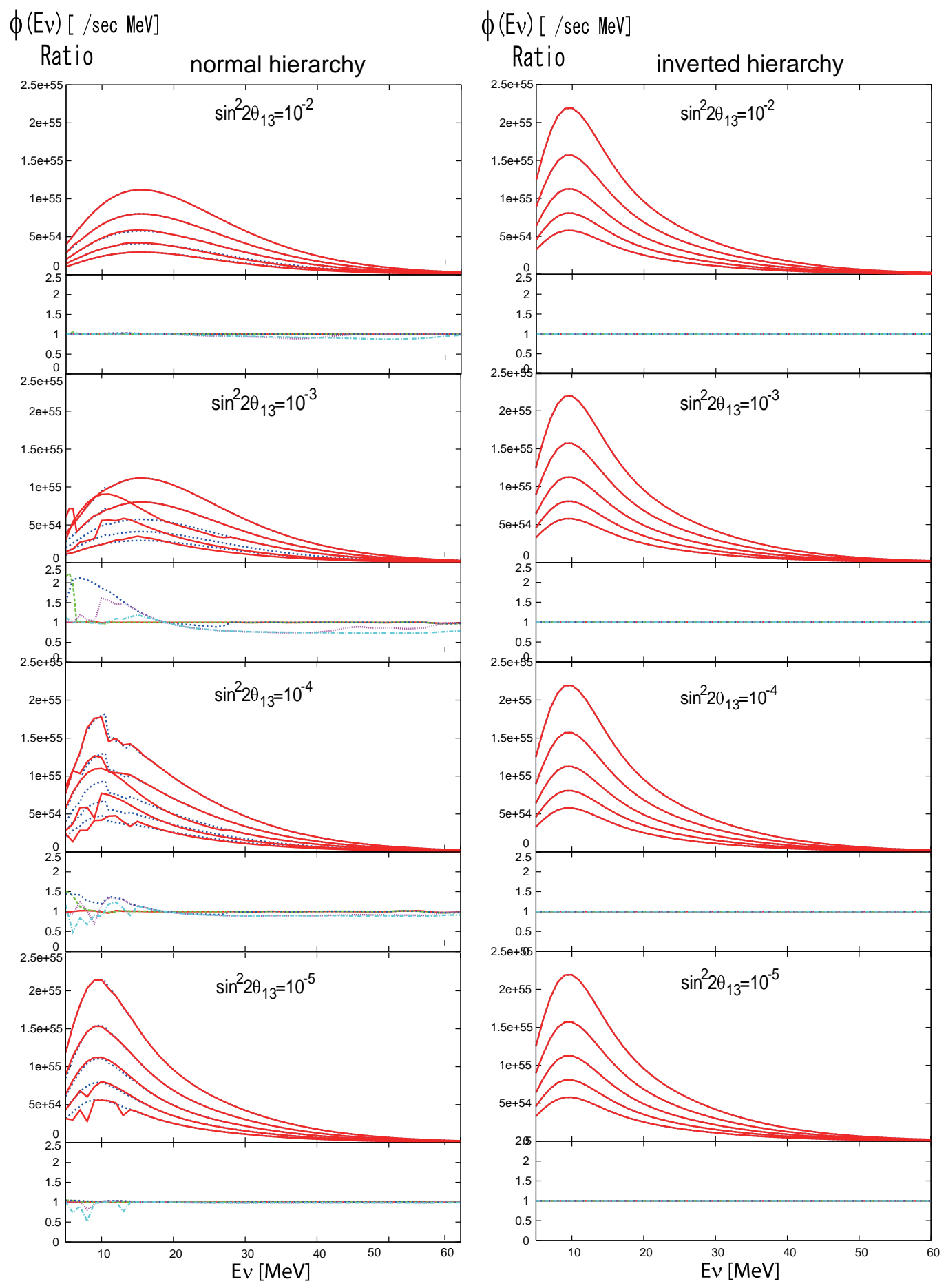

FIG. 4: Upper part of each panels are $\nu_{e}$ spectra $\phi\left(\nu_{e}\right)$ in every 1 second. Solid (red) and dotted (blue) lines are the calculated results with and without shock wave, respectively. Lower part of each panel is the ratio of the spectra with shock to without shock, $\phi_{\text {with }}\left(E_{\nu}\right) / \phi_{\text {without }}\left(E_{\nu}\right)$, at $t=1$ (red), 2(green), 3(blue), 4(purple) and 5(sky blue) sec. The value of $\sin ^{2} 2 \theta_{13}$ is $10^{-2}, 10^{-3}$, $10^{-4}$ and $10^{-5}$ from top to bottom. Left and right panels are in normal and inverted hierarchy, respectively. 


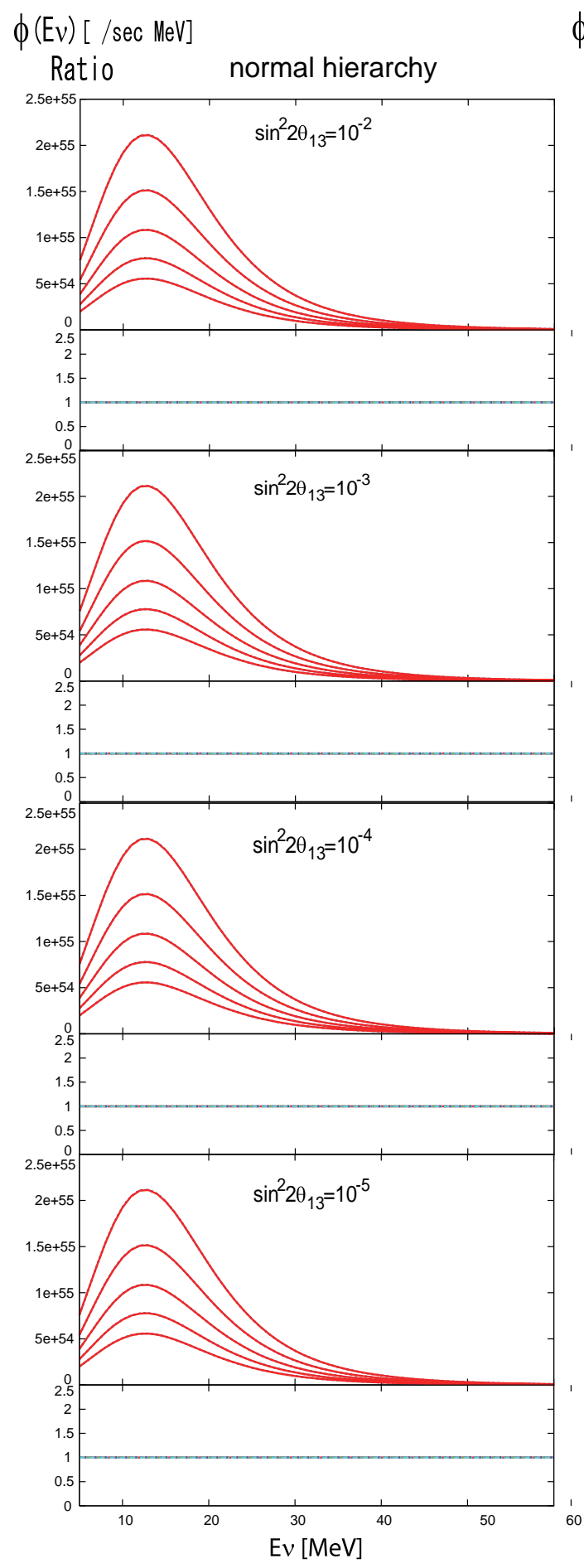

$\phi(E v)$ [ / sec MeV]

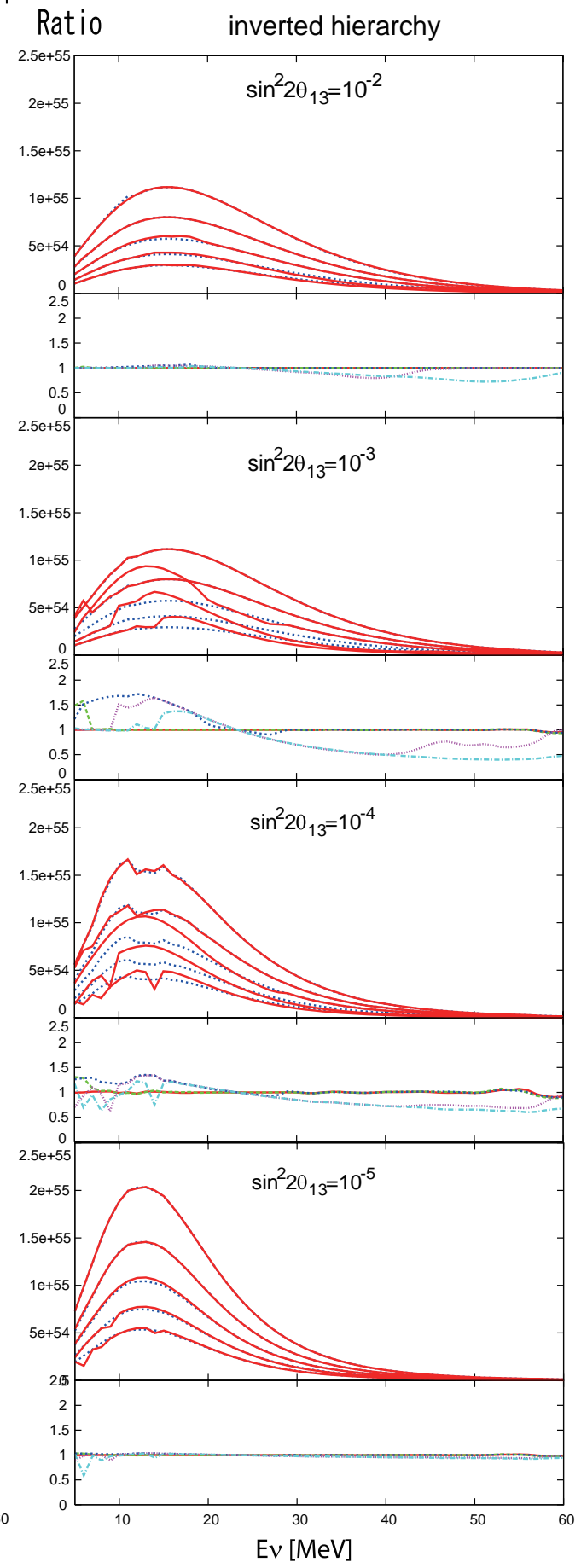

FIG. 5: The same as Figure 4, but for $\bar{\nu}_{e}$ spectra. 

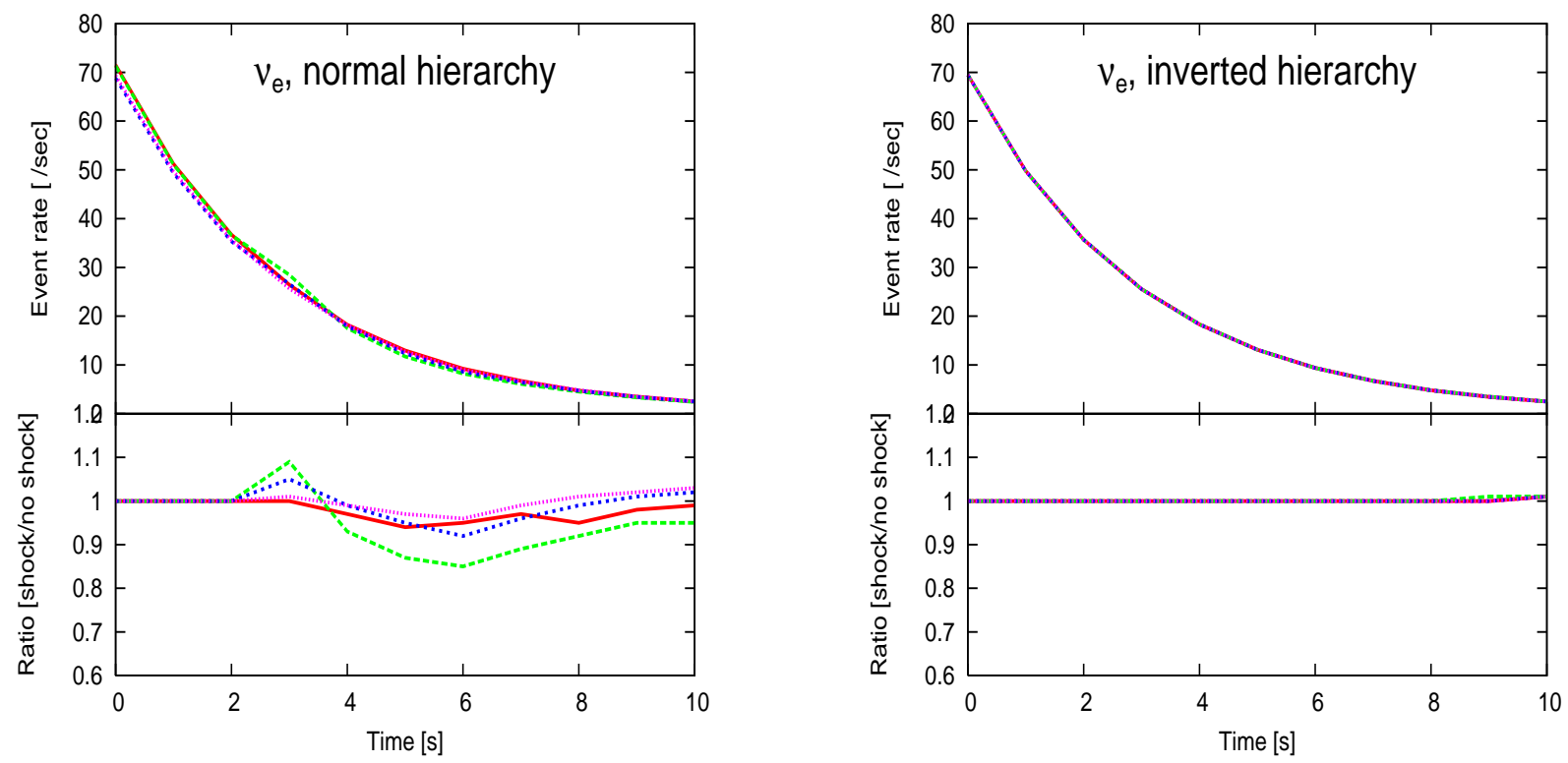

FIG. 6: Upper part of each panels shows the expected energy-integrated event rates of $\nu_{e}$ with SK as a function of time, and lower part shows the ratio of these event rates with shock to those without shock for various mixing angles $\sin ^{2} 2 \theta_{13}=10^{-2}$ (red), $10^{-3}$ (green), $10^{-4}$ (glue) and $10^{-5}$ (purple). Left and right panels are in normal and inverted hierarchy, respectively.
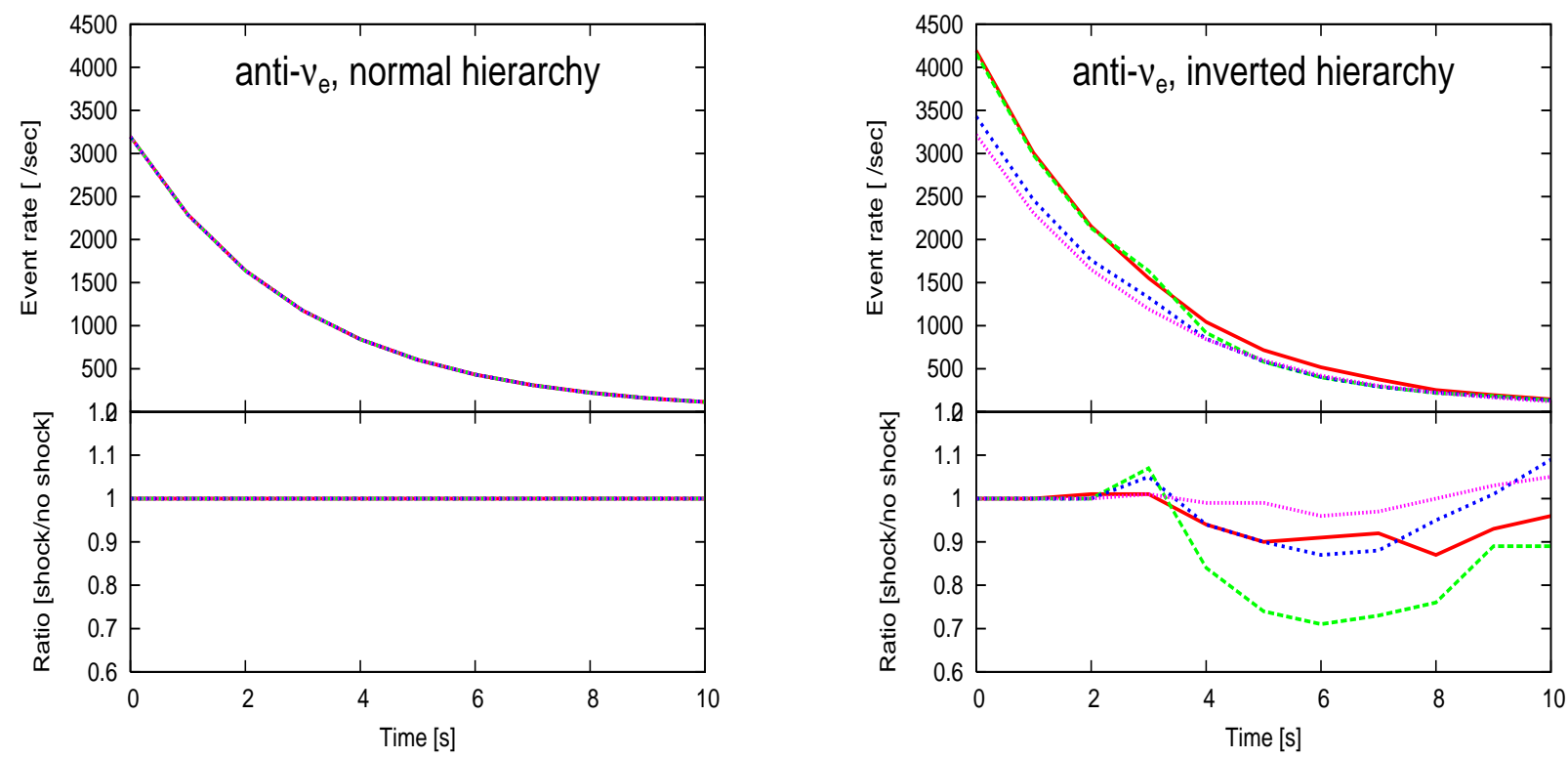

FIG. 7: The same as Figure 6, but for $\bar{\nu}_{e}$. 

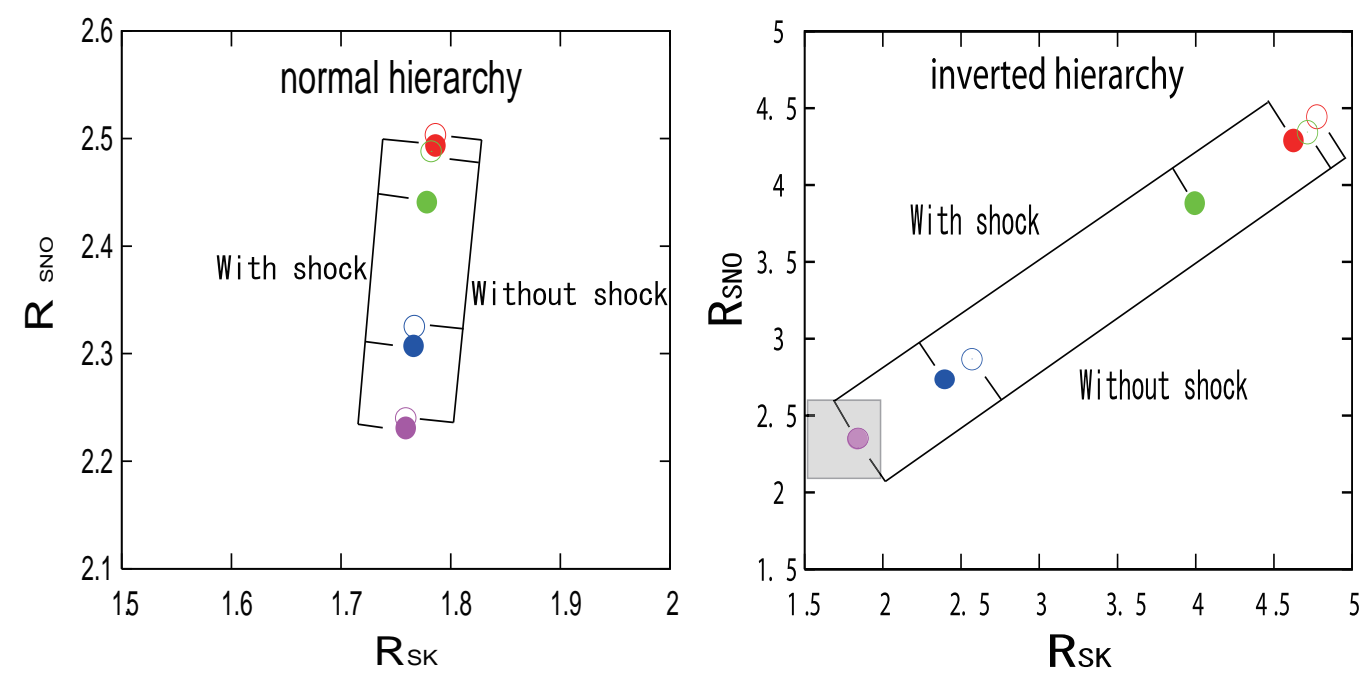

FIG. 8: Calculated correlation between $R_{\mathrm{SK}}$ and $R_{\mathrm{SNO}}$, which are the ratio of the time-integrated events of high-energy neutrinos $\left(20 \mathrm{MeV}<E_{\nu}<60 \mathrm{MeV}\right)$ to those of low-energy neutrinos (5 $\mathrm{MeV}<E_{\nu}<20 \mathrm{MeV}$ ) for the SK and SNO detectors, respectively, for various mixing angles of $\sin ^{2} 2 \theta_{13}=10^{-2}$ (red), $10^{-3}$ (green), $10^{-4}$ (blue) and $10^{-5}$ (purple). Left panel is for normal hierarchy, and right panel for inverted hierarchy. Closed and open circles are the calculated results with and without shock wave, respectively, as denoted. Note that the scale of each panel is different, and a closed square in the right panel corresponds to a range in the left panel. 

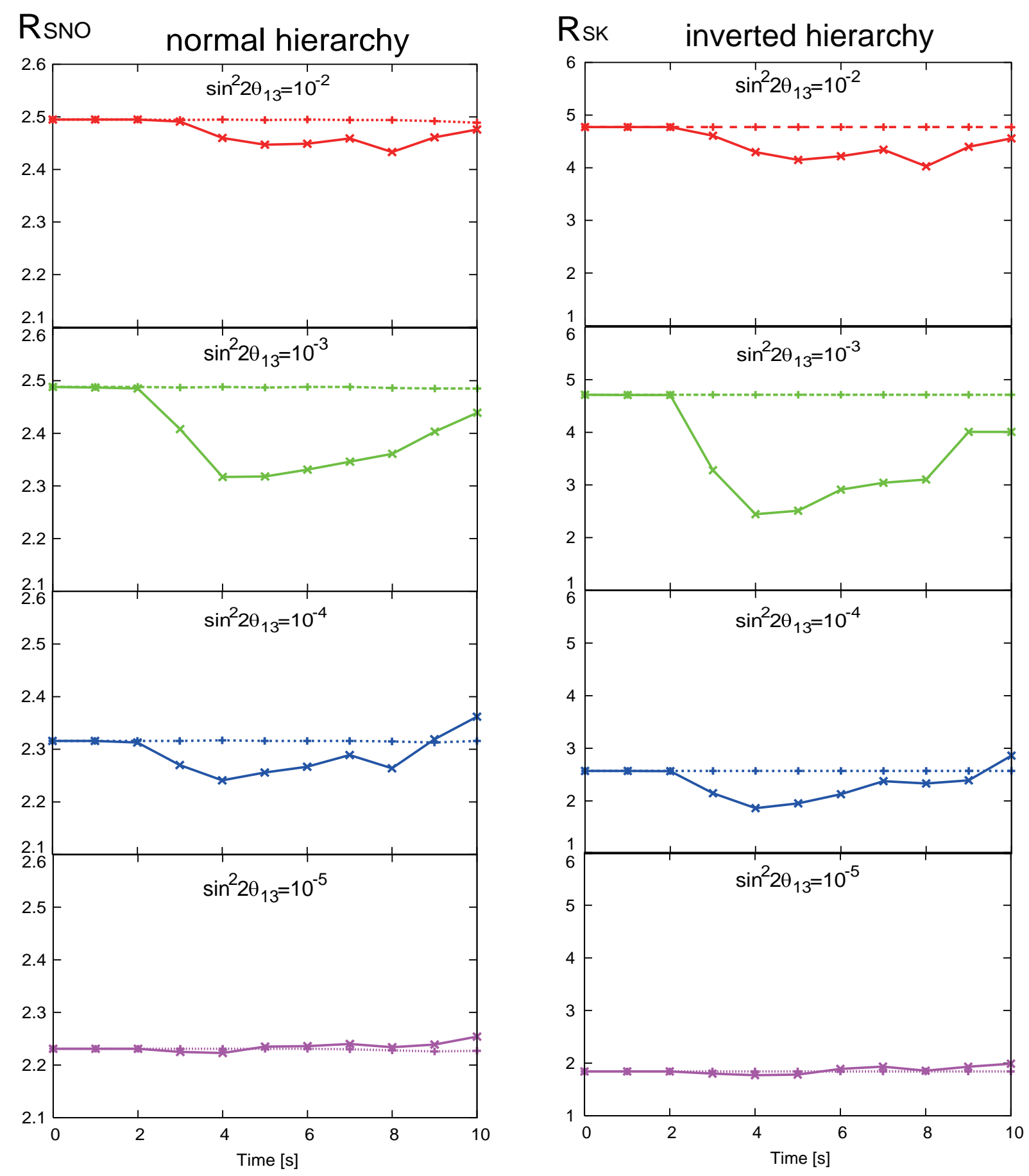

FIG. 9: Time-dependent ratios of the events of high-energy neutrinos $\left(20 \mathrm{MeV}<E_{\nu}<60 \mathrm{MeV}\right)$ to those of low-energy neutrinos $\left(5 \mathrm{MeV}<E_{\nu}<20 \mathrm{MeV}\right)$ for the SK and SNO detectors for various mixing angles of $\sin ^{2} 2 \theta_{13}=10^{-2}, 10^{-3}, 10^{-4}$ and $10^{-5}$ from top to bottom panel. Left panels are the ratio $R_{\mathrm{SNO}}(t)$ for normal hierarchy, and right panels are the ratio $R_{\mathrm{SK}}(t)$ in inverted hierarchy. Solid and dashed lines are the calculated results with and without shock wave, respectively. Note that the scale of each panel is different. 